\title{
Compact representations of partially coherent undulator radiation suitable for wave propagation
}

\author{
Ryan R. Lindberg and Kwang-Je Kim \\ Advanced Photon Source, Argonne National Laboratory, Argonne, Illinois 60439, USA
}

(Received 27 May 2015; published 28 September 2015)

\begin{abstract}
Undulator radiation is partially coherent in the transverse plane, with the degree of coherence depending on the ratio of the electron beam phase space area (emittance) to the characteristic radiation wavelength $\lambda$. On the other hand, numerical codes used to predict x-ray beam line performance can typically only propagate coherent fields from the source to the image plane. We investigate methods for representing partially coherent undulator radiation using a suitably chosen set of coherent fields that can be used in standard wave propagation codes, and discuss such "coherent mode expansions" for arbitrary degrees of coherence. In the limit when the electron beam emittance along at least one direction is much larger than $\lambda$ the coherent modes are orthogonal and therefore compact; when the emittance approaches $\lambda$ in both planes we discuss an economical method of defining the relevant coherent fields that samples the electron beam phase space using low-discrepancy sequences.
\end{abstract}

DOI: 10.1103/PhysRevSTAB.18.090702

PACS numbers: 41.60.Ap

\section{INTRODUCTION}

Modern storage rings now produce partially coherent $\mathrm{x}$ rays for experimental use, and new facilities under both construction and design hold the promise for increasing the coherent flux by $1-2$ orders of magnitude (see, e.g., [1-4]). In tandem with these developments, a host of experimental $\mathrm{x}$-ray techniques have been developed over the last few decades that take advantage of partially coherent radiation, including $x$-ray photon correlation spectroscopy and scattering [5,6], coherent x-ray diffractive imaging [7-9], x-ray scanning microscopy [10], and $\mathrm{x}$-ray nanoprobe spectroscopy [11]. The ability to make detailed predictions of the $\mathrm{x}$-ray properties has become an important component in both the design and interpretation of these experiments. This in turn requires accurately simulating the radiation from the source to the detector, including realistic models of all beam line optical elements, and of the sample itself.

In the limit of either complete incoherence or full coherence, simulation of $\mathrm{x}$-ray propagation through the beam line optics is straightforward: ray tracing can be employed in the former while Fresnel propagation can be applied to the latter. On the other hand, accurately modeling the full coherence properties of partially coherent undulator radiation is a challenging task that can become numerically intensive. In this paper we discuss how partially coherent undulator radiation can be represented optimally for different cases of electron beam distributions typical to both current third generation storage rings and in those of future

Published by the American Physical Society under the terms of the Creative Commons Attribution 3.0 License. Further distribution of this work must maintain attribution to the author $(s)$ and the published article's title, journal citation, and DOI. upgraded machines. Our approach is based on an appropriate summation of coherent fields similar to those discussed in $[12,13]$. This is in contrast to other, approximate methods that are based on judiciously combining raytracing and wavefront propagation; these alternatives have been based on the superposition of Gaussian wavelets [14], the Green function Monte Carlo methods [15], and the socalled "hybrid method" of Ref. [16], but we will not discuss them further. We limit our discussion to the first-order coherence, which can be quantified by the ensemble average of quantities quadratic in electric field amplitudes. Most coherence experiments in synchrotron radiation explore the first-order coherence.

In Sec. II we present some background material covering undulator radiation and its characterization using statistical optics. After beginning with a description of the emission of undulator radiation by a single electron, in Sec. II B we introduce some basic quantities of statistical optics including the cross-spectral density and brightness or Wigner function. We conclude this section with a brief review of a few useful, simple metrics that characterize the coherence that we will apply to undulator radiation later in the paper. Section III presents and compares various ways that partially coherent undulator radiation may be represented using coherent fields or modes, and represents the main results of this paper.

After briefly showing how these "coherent mode" expansions may be used to represent the cross-spectral density, we discuss the simplest such expansion to the undulator field in Sec. III A. This expansion approximates the undulator field by a Gaussian mode, and results in the so-called Gaussian-Schell model of undulator radiation that has a long history in statistical optics. The Gaussian-Schell model only applies if the electron beam phase space area 
(or emittance) in both horizontal and vertical planes is much larger that $\lambda / 4 \pi$. To go beyond this incoherent case, in Sec. III B we investigate the limit where one emittance is large while the other is arbitrary; this case applies at modern third generation storage rings over a wide range of parameters. Here, we find that the Gaussian-Schell expansion is valid along the large-emittance direction, while the coherent modes in the other direction can be found numerically with a matrix eigensolver. Finally, in Sec. III C we turn to the case where the e-beam emittances in both planes can be arbitrarily small. In this case, we show that a relatively compact representation of the field can be built by a suitable Monte Carlo sampling of the electron beam phase space with low-discrepancy sequences. Finally, we conclude in Sec. IV.

\section{STATISTICAL OPTICS OF UNDULATOR RADIATION}

Undulator radiation is most naturally described using statistical optics. This section is primarily a review of the necessary concepts and an introduction to our notation. We begin with the paraxial description of the field, and proceed to write down the radiation generated by a reference electron moving along the optical axis in an undulator. We then relate the reference solution to that of an electron with arbitrary initial coordinates in phase space. Since the particle coordinates are only known probabilistically, we are naturally led to characterize the field statistically. We then briefly present some basic notions from statistical optics, and show how a specific correlation function (the cross-spectral density) describes undulator radiation.

\section{A. Paraxial description of undulator radiation}

Within the paraxial approximation, the electric field $E(\boldsymbol{x}, t ; z)$ is represented at the location $z$ along the optical axis as a function of the time $t$ and the coordinates $\boldsymbol{x}=(x, y)$ in the plane transverse to $z$. We will find it convenient to write the field in terms of its Fourier transform with respect to both $t$ and $\boldsymbol{x}$, and therefore introduce the angular-spectral representation of the field

$$
\tilde{E}_{k}(\boldsymbol{\phi} ; z) \equiv \frac{1}{2 \pi \lambda^{2}} \int d \boldsymbol{x} d t E(\boldsymbol{x}, t ; z) e^{i k(c t-\boldsymbol{x} \cdot \boldsymbol{\phi})},
$$

where $k=2 \pi / \lambda=\omega / c$ is the $\hat{z}$ component of the wave vector and $|\boldsymbol{\phi}| \ll 1$ is the angle with respect to the optical axis. The evolution of the field $\tilde{E}_{k}$ is governed by the paraxial wave equation

$$
\begin{aligned}
& {\left[\frac{\partial}{\partial z}+\frac{i k}{2} \boldsymbol{\phi}^{2}\right] \tilde{\boldsymbol{E}}_{k}(\boldsymbol{\phi} ; z)} \\
& \quad=-\int d t d \boldsymbol{x} \frac{\boldsymbol{J}-c \rho \boldsymbol{\phi}}{4 \pi \epsilon_{0} c \lambda^{2}} e^{i k(c t-z-\boldsymbol{\phi} \cdot \boldsymbol{x})},
\end{aligned}
$$

where $\epsilon_{0}$ is the permittivity of free space, $c$ is the speed of light, and $\rho, \boldsymbol{J}$ are the charge and current density, respectively. For synchrotron radiation produced by highly relativistic electrons whose energy $\gamma \gg 1$ and longitudinal velocity $d z / d t \approx c$, the source terms due to electron $j$ are given by

$$
\begin{aligned}
& \rho_{j}(\boldsymbol{x}, t, z)=-\frac{e}{c} \delta\left[\boldsymbol{x}-\boldsymbol{x}_{j}(z)\right] \delta\left[t-t_{j}(z)\right], \\
& \boldsymbol{J}_{j}(\boldsymbol{x}, t, z)=c \boldsymbol{x}_{j}^{\prime} \rho_{j}(\boldsymbol{x}, t, z)
\end{aligned}
$$

where $e$ is the magnitude of the electron charge and $\boldsymbol{x}_{j}^{\prime} \equiv$ $d \boldsymbol{x}_{j} / d z$ is the angle that the electron makes with the optical axis $z$. We will consider synchrotron radiation from an undulator whose period and peak magnetic field are $\lambda_{u}$ and $B_{0}$, respectively. We introduce the reference electron energy $\gamma_{r}$, which defines the fundamental radiation wavelength $\lambda_{r}=2 \pi / k_{r}$ in terms of the undulator period $\lambda_{u}=$ $2 \pi / k_{u}$ and deflection parameter $K \equiv e B_{0} / m c k_{u}$ via

$$
\lambda_{r} \equiv \frac{1+K^{2} / 2}{2 \gamma_{r}^{2}} \lambda_{u}
$$

We model the undulator as an ideal, hard-edged device of length $L_{u}$ that is centered at $z=0$, assuming that the magnetic field is sinusoidal and directed along $\hat{\boldsymbol{y}}$ when $|z| \leq L_{u} / 2=N_{u} \lambda_{u} / 2$ and zero otherwise $\left(N_{u}\right.$ is the number of undulator periods). If we assume further that the electron energy deviation from resonance is small, $\left|\eta_{j}\right| \equiv$ $\left|\gamma_{j}-\gamma_{r}\right| / \gamma_{r} \ll 1$, and that the natural undulator focusing is negligible, $2 \pi K N_{u} / \gamma_{r} \ll 1$, then the electron trajectory follows the wiggle motion in $x$ and a simple straight line along $y$ :

$$
\begin{aligned}
& x_{j}^{\prime}(z)=\frac{K \cos \left(k_{u} z\right)}{\gamma_{r}}+x_{j}^{\prime}, \quad y_{j}^{\prime}(z)=y_{j}^{\prime}, \\
& x_{j}(z)=\frac{K \sin \left(k_{u} z\right)}{\gamma_{r} k_{u}}+x_{j}^{\prime} z+x_{j}, \quad y_{j}(z)=y_{j}^{\prime} z+y_{j} .
\end{aligned}
$$

Here, we have defined the "initial" coordinates $\boldsymbol{x}_{j} \equiv \boldsymbol{x}_{j}(0)$ and $\boldsymbol{x}_{j}^{\prime} \equiv \boldsymbol{x}_{j}^{\prime}(0)$ to be those that the particle would have at $z=0$ in the absence of the undulator; the ideal reference electron has $\boldsymbol{x}_{j}=\boldsymbol{x}_{j}^{\prime}=\mathbf{0}$. The electron time coordinate under the same assumptions is

$$
\begin{aligned}
c t_{j}(z)= & {\left[1+\frac{k_{u}}{k_{r}}\left(1-2 \eta_{j}\right)\right] z+\frac{K}{4 \gamma_{r}^{2} k_{u}} \sin \left(2 k_{u} z\right) } \\
& +\frac{K x_{j}^{\prime}}{\gamma_{r} k_{u}} \sin \left(k_{u} z\right)+\frac{1}{2} x_{j}^{\prime 2} z+c t_{j},
\end{aligned}
$$


where $t_{j}=t_{j}(0)$ is the time when the particle passes the plane $z=0$ and the reference electron has $t_{j}=0$.

We can now determine the electric field by inserting the particle trajectories (6)-(8) into the sources (3) and (4), which we then use in the paraxial wave equation (2). While a complete solution can be easily written down, we will focus on the field within the central cone defined by $\gamma_{r}|\boldsymbol{\phi}| \lesssim 1 / \sqrt{\left(2+K^{2}\right) N_{u}}$. In this case the source dramatically simplifies, particularly for the reference electron whose initial phase space coordinates vanish; the undulator field in the central cone due to this reference electron is

$$
\begin{aligned}
\tilde{E}_{k}^{0}(\boldsymbol{\phi} ; 0)= & \frac{e K[\mathrm{JJ}] L_{u}}{8 \pi \epsilon_{0} \gamma_{r} c \lambda^{2}} e^{-i \pi N_{u}\left(k-k_{r}\right) / k_{r}} \\
& \times \operatorname{sinc}\left[\pi N_{u}\left(\frac{k_{r}}{2 k_{u}} \boldsymbol{\phi}^{2}+\frac{k-k_{r}}{k_{r}}\right)\right],
\end{aligned}
$$

where we use the standard notation for the Bessel function factor $[\mathrm{JJ}] \equiv J_{0}\left[\frac{K^{2}}{4+2 K^{2}}\right]-J_{1}\left[\frac{K^{2}}{4+2 K^{2}}\right]$. Equation (9) expresses the field at the virtual source point $z=0$ (i.e., in the middle of the undulator), which is what one would measure if the field was imaged downstream with 1:1 optics.

Before continuing, we will find it convenient to introduce dimensionless coordinates. As seen in (9), the natural angular divergence $\sim \sqrt{\lambda_{r} / L_{u}}$, which implies a diffraction limited waist $\sim \sqrt{L_{u} \lambda_{r}}$. While the precise scaling is somewhat arbitrary, we choose to define dimensionless transverse coordinates in terms of the rms angular and spatial widths $\sigma_{r^{\prime}}$ and $\sigma_{r}$ as

$$
\begin{gathered}
\hat{\boldsymbol{\phi}} \equiv \frac{\boldsymbol{\phi}}{\sqrt{2} \sigma_{r^{\prime}}} \quad \text { with } \quad \sigma_{r^{\prime}} \equiv \frac{1}{2} \sqrt{\frac{\lambda_{r}}{L_{u}}} \\
\hat{\boldsymbol{x}} \equiv \frac{\boldsymbol{x}}{\sqrt{2} \sigma_{r}} \quad \text { with } \quad \sigma_{r} \equiv \frac{\sqrt{\lambda_{r} L_{u}}}{2 \pi}
\end{gathered}
$$

Note that the product $\sigma_{r} \sigma_{r^{\prime}}$ equals the minimum radiation phase space area $\lambda_{r} / 4 \pi$, while the coordinate scaling was chosen such that $e^{i k_{r} \boldsymbol{\phi} \cdot \boldsymbol{x}}=e^{i \hat{\boldsymbol{\phi} \cdot \hat{x}}}$; we will explain our rationale for the precise values of $\sigma_{r}$ and $\sigma_{r^{\prime}}$ shortly. Before that, we define the scaled frequency $\nu \equiv k / k_{r}$ and the dimensionless frequency detuning from resonance $\delta_{\nu} \equiv \pi N_{u}(\nu-1)$, in terms of which the undulator field

$$
\tilde{E}_{k}^{0}(\boldsymbol{\phi} ; 0)=\frac{e K[\mathrm{JJ}] L_{u}}{8 \pi \epsilon_{0} \gamma_{r} c \lambda^{2}} e^{-i \delta_{\nu}} \operatorname{sinc}\left(\frac{\pi}{4} \hat{\boldsymbol{\phi}}^{2}+\delta_{\nu}\right) .
$$

Finally, we introduce the dimensionless paraxial field produced by the reference electron as

$$
\mathcal{E}_{\nu}^{0}(\hat{\boldsymbol{\phi}} ; 0) \equiv \frac{8 \pi \epsilon_{0} \gamma_{r} c \lambda^{2}}{e K[\mathrm{JJ}] L_{u}} e^{i \delta_{\nu}} \tilde{E}_{k}^{0}=\operatorname{sinc}\left(\frac{\pi}{4} \hat{\boldsymbol{\phi}}^{2}+\delta_{\nu}\right)
$$

We now comment on the chosen values of the rms size (11) and divergence (10), which depend on how one fits the undulator field (13) to a Gaussian. If we were to match the maximum of $\mathcal{E}_{\nu}^{0}$ and integrated flux $\int d \hat{\boldsymbol{\phi}}|\mathcal{E}(\hat{\boldsymbol{\phi}})|^{2}$, we would find that $\sigma_{r}^{(1)}=\sqrt{2 L_{u} \lambda_{1}} / 4 \pi$ and $\sigma_{r^{\prime}}^{(1)}=\sqrt{\lambda_{1} / 2 L_{u}}$; this was first derived in [17], from which the effective Rayleigh range of undulator radiation was determined to be $Z_{R}^{(1)}=\sigma_{r}^{(1)} / \sigma_{r^{\prime}}^{(1)}=L_{u} / 2 \pi$. However, in the spatial domain this approximation results in a Gaussian peak intensity that is four times higher than that obtained from Fourier transforming (13). On the other hand, if we chose our Gaussian to match the flux and the peak of the spatial field we would find that $\sigma_{r^{\prime}}^{(2)}=\sqrt{\lambda_{1} / 8 L_{u}}, \sigma_{r}^{(2)}=\sqrt{2 \lambda_{1} L_{u}} / 2 \pi$ and $Z_{R}^{(2)}=2 L_{u} / \pi$. The choice for the rms field size and divergence in Eqs. (10) and (11) splits the difference between the two predictions described above, so that the corresponding Gaussian approximation has the same flux but twice the peak intensity of the undulator radiation in both angular and spatial representations. The resulting effective Rayleigh range of the undulator field is $Z_{R}=\sigma_{r} / \sigma_{r^{\prime}}=L_{u} / \pi$.

The reference field in (13) applies to the reference electron whose initial phase space coordinates $\left(\hat{\boldsymbol{x}}_{j}, \hat{\boldsymbol{x}}_{j}^{\prime}, t_{j}, \eta_{j}\right)$ are all identically zero. Nevertheless, $\mathcal{E}_{\nu}^{0}$ can be simply related to the field $\mathcal{E}_{\nu}$ from a generic electron by considering how the source terms in the paraxial equation change for nonzero initial coordinates $\left(\boldsymbol{x}_{j}, \boldsymbol{x}_{j}^{\prime}, \eta_{j}, t_{j}\right)$. We find that [18]

$$
\mathcal{E}_{\nu}(\hat{\boldsymbol{\phi}} ; 0)=e^{i c k t_{j}} e^{-i \hat{\boldsymbol{\phi}} \cdot \hat{\boldsymbol{x}}_{j}} \mathcal{E}_{\nu-2 \eta_{j}}^{0}\left(\hat{\boldsymbol{\phi}}-\hat{\boldsymbol{x}}_{j}^{\prime} ; 0\right)
$$

where $\hat{\boldsymbol{x}}_{j} \equiv \boldsymbol{x} \sqrt{2} / \sigma_{r}$ and $\hat{\boldsymbol{x}}_{j}^{\prime} \equiv \boldsymbol{x}_{j}^{\prime} \sqrt{2} / \sigma_{r^{\prime}}$. The result (14) reflects the fact that an initial offset in $\hat{\boldsymbol{x}}_{j}\left(\hat{\boldsymbol{x}}_{j}^{\prime}\right)$ effectively displaces the optical axis in position (angle), while different values of $t_{j}$ and $\eta_{j}$ give rise to shifts in the time and resonance condition.

As indicated by (14), the total radiation field produced by a collection of electrons will depend on the particle microdistribution in phase space. Since we only directly know certain statistical properties regarding the coordinates (typically the first and second moments of a nearly Gaussian distribution), it is appropriate to describe undulator radiation using the formalism of statistical optics. In the next section we review some of the relevant physics as applied to synchrotron emission. 


\section{B. Undulator coherence: Cross spectral density, mutual coherence, brightness, and all that}

The statistics of undulator radiation are those of a Gaussian random process as explained in [19], so that all field correlations can be derived from the second order correlation function (see [20] for some more general discussion). The two-point spectral-angular correlation function of the electric field is

$$
\tilde{\mathcal{C}}\left(\hat{\boldsymbol{\phi}}_{1}, \nu_{1}, \hat{\boldsymbol{\phi}}_{2}, \nu_{2} ; z\right) \propto\left\langle\mathcal{E}_{\nu_{1}}\left(\hat{\boldsymbol{\phi}}_{1} ; z\right) \mathcal{E}_{\nu_{2}}\left(\hat{\boldsymbol{\phi}}_{2} ; z\right)^{*}\right\rangle,
$$

where the angular brackets denote an ensemble average over the initial positions, angles, energies, and arrival times of the radiating electrons. For typical parameters of storage rings, $\tilde{\mathcal{C}}$ is separable into its spectral and spatial parts [19]. In particular, if we assume that the electron bunch is Gaussian with temporal width $\sigma_{\tau}$ that is much longer than the undulator field coherence time, $\sigma_{\tau} \gg N_{u} \lambda_{r} / c$, then the spectral coherence is dictated by the electron beam; for typical storage rings $\sigma_{\tau} \gtrsim 10 \mathrm{ps}$ and $N_{u} \lesssim 300$, which restricts the wavelengths of interest to $\lambda_{r} \ll 10 \mu \mathrm{m}$. Hence, for $\mathrm{x}$ rays we can write

$$
\tilde{\mathcal{C}} \approx e^{-\left[c \sigma_{\tau} k_{r}\left(\nu_{1}-\nu_{2}\right)\right]^{2} / 2} \tilde{W}_{\nu}\left(\hat{\boldsymbol{\phi}}_{1}, \hat{\boldsymbol{\phi}}_{2} ; z\right),
$$

where the average scaled frequency $\nu=\left(\nu_{1}+\nu_{2}\right) / 2 \approx$ $\nu_{1} \approx \nu_{2}$, and we have separated out the cross-spectral density $\tilde{W}_{\nu}$; at the source point this is given by

$$
\begin{aligned}
\tilde{W}_{\nu}\left(\hat{\boldsymbol{\phi}}_{1}, \hat{\boldsymbol{\phi}}_{2} ; 0\right) \equiv & \left\langle\frac{\mathcal{F}_{1}}{(4 \pi)^{2}} \sum_{j=1}^{N_{e}} \mathcal{E}_{\nu}\left(\hat{\boldsymbol{\phi}}_{1} ; 0\right) \mathcal{E}_{\nu}\left(\hat{\boldsymbol{\phi}}_{2} ; 0\right)^{*}\right\rangle \\
& +\left\langle\sum_{j \neq \ell} e^{i c k_{\nu} \nu\left(t_{j}-t_{e}\right)}[\cdots]\right\rangle .
\end{aligned}
$$

Here, the total number of electrons is $N_{e}$, and we have introduced the single electron resonant flux $\mathcal{F}_{i}$. In terms of the fine structure constant $\alpha \equiv e^{2} /\left(4 \pi \epsilon_{0} \hbar c\right)$ ( $\hbar$ is Planck's constant divided by $2 \pi$ ) and the averaging time $T$, the single electron resonant flux is

$$
\mathcal{F}_{1} \equiv \frac{\pi \alpha N_{u}}{2 T} \frac{K^{2}[\mathrm{JJ}]^{2}}{\left(1+K^{2} / 2\right)}
$$

In addition, we have normalized (17) such that for a monoenergetic beam the first line comprises the flux

$$
\begin{aligned}
N_{e} \mathcal{F}_{\nu} & =\int d \hat{\boldsymbol{\phi}} \tilde{W}_{\nu}(\hat{\boldsymbol{\phi}}, \hat{\boldsymbol{\phi}} ; z) \\
& =N_{e} \mathcal{F}_{1}\left\{1-\frac{2}{\pi}\left[\operatorname{Si}\left(\delta_{\nu}\right)-\frac{\sin ^{2}\left(\delta_{\nu} / 2\right)}{\delta_{\nu} / 2}\right]\right\},
\end{aligned}
$$

where $\operatorname{Si}(x) \equiv \int_{0}^{x} d t \sin t / t$ is the sine integral of $x$.
We can neglect the second line from the cross-spectral density (17) if the electron beam does not have significant Fourier content (bunching) at the wavelength of interest, in which case the sum over random phases gives a negligibly small contribution. For our Gaussian electron bunch dropping the second line in (17) is valid for wavelengths shorter than the bunch length $\lambda<c \sigma_{\tau}$, which is automatically satisfied under the condition $\lambda \ll c \sigma_{\tau} / N_{u}$ that was assumed to separate the correlation function according to (16).

We compute the ensemble average associated with the cross-spectral density (17) using the single particle probability function $f_{e}\left(\hat{\boldsymbol{x}}_{j}, \hat{\boldsymbol{x}}_{j}^{\prime}, \eta_{j}\right)$ assuming that the particle coordinates are uncorrelated and independent. We normalize $f_{e}$ such that integrating over all coordinates is unity, and further assume that this distribution is separable into its energy and transverse parts, $f_{e}\left(\hat{\boldsymbol{x}}_{j}, \hat{\boldsymbol{x}}_{j}^{\prime}, \eta_{j}\right)=g\left(\eta_{j}\right) f\left(\hat{\boldsymbol{x}}_{j}, \hat{\boldsymbol{x}}_{j}^{\prime}\right)$. In this case, the cross-spectral density can be written as

$\tilde{W}_{\nu}\left(\hat{\boldsymbol{\phi}}_{1}, \hat{\boldsymbol{\phi}}_{2} ; 0\right)=\frac{N_{e} \mathcal{F}_{1}}{(4 \pi)^{3}} \int d \eta g(\eta) \mathcal{W}_{\nu-2 \eta}\left(\hat{\boldsymbol{\phi}}_{1}, \hat{\boldsymbol{\phi}}_{2} ; z\right)$

with

$$
\begin{aligned}
\mathcal{W}_{\nu}\left(\hat{\boldsymbol{\phi}}_{1}, \hat{\boldsymbol{\phi}}_{2} ; 0\right)= & \int d \hat{\boldsymbol{x}} d \hat{\boldsymbol{x}}^{\prime} \frac{f\left(\hat{\boldsymbol{x}}, \hat{\boldsymbol{x}}^{\prime}\right)}{2 \pi} e^{-i\left(\hat{\boldsymbol{\phi}}_{1}-\hat{\boldsymbol{\phi}}_{2}\right) \cdot \hat{\boldsymbol{x}}} \\
& \times \mathcal{E}_{\nu}^{0}\left(\hat{\boldsymbol{\phi}}_{1}-\hat{\boldsymbol{x}}^{\prime} ; 0\right) \mathcal{E}_{\nu}^{0}\left(\hat{\boldsymbol{\phi}}_{2}-\hat{\boldsymbol{x}}^{\prime} ; 0\right)^{*}
\end{aligned}
$$

Note that $\int d \boldsymbol{\phi} \mathcal{W}_{\nu}(\boldsymbol{\phi}, \boldsymbol{\phi} ; 0)=\mathcal{F}_{\nu} / \mathcal{F}_{1}$.

We have shown that at the source $\tilde{W}_{\nu}\left(\hat{\boldsymbol{\phi}}_{1}, \hat{\boldsymbol{\phi}}_{2} ; 0\right)$ can be written as a convolution of the electron energy distribution with the reduced cross-spectral density $\mathcal{W}_{\nu}$. In addition, beam lines composed of optical elements that treat each frequency component separately preserve the division shown in (20) and (21) to the output plane. Most X-ray optical elements work this way, including apertures, free space propagation, reflection and transmission from mirrors and lenses with or without abberations, and most x-ray crystal optics such as monochromators based on Bragg or Laue diffraction. Hence, if one can find a way to propagate the relevant frequency components of $\mathcal{W}_{\nu}$ through the beam line, in most situations the final cross-spectral density can be found by convolving $\mathcal{W}_{\nu}$ at the output plane with the electron beam energy distribution. The primary goal of this paper is to discuss a simple method by which $\mathcal{W}_{\nu}$ can be transported through an optical system.

In principle one can write down evolution operators for $\mathcal{W}_{\nu}$ itself. As long as the propagation is not described statistically (as might be the case for some models of element vibrations or mirror surface microroughness), the evolution of $\mathcal{W}_{\nu}$ derives directly from the evolution of the field $\mathcal{E}_{\nu}$. However, computing and then manipulating the fourdimensional $\mathcal{W}_{\nu}$ is a nontrivial computational task. Moreover, there has been considerable effort and progress in developing wave optics simulation codes designed to 
propagate coherent fields down realistic beam lines (see, e.g., [21-23]). It would be nice if one could use such simulation tools to also propagate partially coherent light such as undulator radiation. The next section will discuss how one can construct certain "coherent mode" representations of $\mathcal{W}_{\nu}$ that can be propagated in just such a manner.

Before doing that, we want to mention a few other quantities from statistical optics that we will find useful. First, while it is simplest to describe the propagation of $\mathcal{W}_{\nu}$, it is often convenient to visualize the coherence function in terms of the average coordinate $\hat{\boldsymbol{\phi}} \equiv\left(\hat{\boldsymbol{\phi}}_{1}+\hat{\boldsymbol{\phi}}_{2}\right) / 2$ and the coordinate difference $\hat{\boldsymbol{\xi}} \equiv \hat{\boldsymbol{\phi}}_{1}-\hat{\boldsymbol{\phi}}_{2}$. This defines the crosscorrelation function via

$$
\tilde{\Gamma}_{\nu}(\boldsymbol{\phi}, \boldsymbol{\xi} ; z)=\mathcal{W}_{\nu}\left(\boldsymbol{\phi}+\frac{1}{2} \boldsymbol{\xi}, \boldsymbol{\phi}-\frac{1}{2} \xi ; z\right)
$$

Next, we will want some straightforward methods to characterize the source strength and coherence. The latter can be done using the degree of spectral coherence $\gamma_{\nu}\left(\hat{\boldsymbol{x}}_{1}, \hat{\boldsymbol{x}}_{2}\right)$. As our notation suggests, the normalized coherence function $\gamma_{\nu}$ is most naturally defined in the spatial domain, since it is directly related to the visibility of coherence fringes from two pinholes placed at $\hat{\boldsymbol{x}}_{1}$ and $\hat{\boldsymbol{x}}_{2}$. The degree of spectral coherence is defined as

$$
\gamma_{\nu}\left(\hat{\boldsymbol{x}}_{1}, \hat{\boldsymbol{x}}_{2} ; z\right) \equiv \frac{\Gamma_{\nu}\left(\hat{\boldsymbol{x}}_{1}, \hat{\boldsymbol{x}}_{2} ; z\right)}{\sqrt{I_{\nu}\left(\hat{\boldsymbol{x}}_{1} ; z\right) I_{\nu}\left(\hat{\boldsymbol{x}}_{2} ; z\right)}},
$$

where the intensity $I_{\nu}(\hat{\boldsymbol{x}} ; z) \equiv \Gamma_{\nu}(\hat{\boldsymbol{x}}, \hat{\mathbf{0}} ; z)$, and we hope to make clear that here $\Gamma_{\nu}$ is evaluated in physical space by keeping the arguments $\hat{\boldsymbol{x}}_{1,2}$. The definition (23) implies that fully coherent light has $\left|\gamma_{\nu}\left(\hat{\boldsymbol{x}}_{1}, \hat{\boldsymbol{x}}_{2} ; z\right)\right|=1$ for all pairs $\left(\hat{\boldsymbol{x}}_{1}, \hat{\boldsymbol{x}}_{2}\right)$, while $\gamma_{\nu}=0$ describes a complete lack of coherence with no interference fringes.

Finally, our discussion would not be complete without mentioning the brightness function $\mathcal{B}_{\nu}(\hat{\boldsymbol{\phi}}, \hat{\boldsymbol{x}})$, which plays the role of a (quasi)distribution function for the radiation on the angle-position phase space. $\mathcal{B}_{\nu}$ is defined as the ensemble averaged Wigner function associated with $\mathcal{E}_{\nu}$, which can be shown to equal the Fourier transform of the cross-correlation function:

$$
\mathcal{B}_{\nu}(\hat{\boldsymbol{\phi}}, \hat{\boldsymbol{x}} ; z) \equiv \frac{1}{(2 \pi)^{2}} \int d \hat{\boldsymbol{\xi}} e^{i \hat{\xi} \cdot \hat{x}} \tilde{\Gamma}_{\nu}(\boldsymbol{\phi}, \boldsymbol{\xi} ; z)
$$

For ideal optical elements, the brightness function as defined in Eq. (24) transforms in the same manner as does the phase space distribution of geometrical optics [18]. Hence, the brightness is a physically appealing representation of second-order coherence, and it has recently received renewed interest [24-26].

The brightness can be used to define the source strength and purity in a number of ways. For example, Bastiaans
[27] advocates using the informational or Shannon entropy to characterize coherence, which was more deeply described in [28]; another definition for the degree of coherence or mode purity expresses the number of transverse modes $M_{T}$ by

$$
\frac{1}{M_{T}}=\frac{\int d \hat{\boldsymbol{\phi}} d \hat{\boldsymbol{x}}\left[2 \pi \mathcal{B}_{\nu}(\hat{\boldsymbol{\phi}}, \hat{\boldsymbol{x}})\right]^{2}}{\left[\int d \hat{\boldsymbol{\phi}} d \hat{\boldsymbol{x}} \mathcal{B}(\hat{\boldsymbol{\phi}}, \hat{\boldsymbol{x}} ; z)\right]^{2}} .
$$

Yet another metric characterizing the source strength employs the value of the brightness along the optical axis [17]; $\mathcal{B}_{\nu}(\mathbf{0}, \mathbf{0} ; z)$ is conserved by propagation through free space and ideal thin lenses and mirrors, and represents the maximum "photon density." Dividing the brightness at the origin by the total flux gives another measure of the mode purity,

$$
\begin{aligned}
\mathscr{B}_{0} & \equiv \frac{\pi^{2} \mathcal{F}_{1}}{\mathcal{F}_{\nu}} \mathcal{B}_{\nu}(\mathbf{0}, \mathbf{0} ; z) \\
& =\frac{\mathcal{F}_{1}}{4 \mathcal{F}_{\nu}} \int d \hat{\boldsymbol{\xi}} \mathcal{W}_{\nu}\left(\frac{1}{2} \hat{\boldsymbol{\xi}},-\frac{1}{2} \hat{\boldsymbol{\xi}} ; z\right) .
\end{aligned}
$$

It can be shown that $0 \leq \mathscr{B}_{0} \leq 1$, and that when $\mathscr{B}_{0}$ is unity the source is largely coherent; in terms of the pinhole experiment, $\mathscr{B}_{0}=1$ implies that pinholes placed symmetrically about the optical axis will observe interference fringes with maximal visibility, $\gamma_{\nu}(\boldsymbol{x},-\boldsymbol{x})=1$.

\section{REPRESENTING THE CROSS-SPECTRAL DENSITY WITH COHERENT MODES}

Suppose that we can represent $\mathcal{W}_{\nu}$ at the source point as a weighted "incoherent sum" of the cross-spectral densities associated with the fields $\psi_{n}$,

$$
\mathcal{W}_{\nu}\left(\hat{\boldsymbol{\phi}}_{1}, \hat{\boldsymbol{\phi}}_{2} ; 0\right)=\sum_{n} \kappa_{n} \psi_{n}\left(\hat{\boldsymbol{\phi}}_{1} ; 0\right) \psi_{n}\left(\hat{\boldsymbol{\phi}}_{2} ; 0\right)^{*},
$$

where the constant weights $\kappa_{n}$ are determined by $\mathcal{W}_{\nu}$ and the field set $\{\psi\}$. Then, as long as the evolution of the electric field commutes with the ensemble average, we can propagate the (coherent) fields $\psi_{n}$ using standard wave optics to the output plane, at which point the cross-spectral density $\mathcal{W}_{\nu}$ can be constructed by summing the fields in a manner similar to that of (27). To be more concrete, if the field propagation can be written as a general linear operator $\mathcal{E}_{\nu}(z)=\mathcal{U}\left[\mathcal{E}_{\nu}(0)\right]$ with the property that $\langle\mathcal{U}[\mathcal{E}]\rangle=\mathcal{U}[\langle\mathcal{E}\rangle]$, then it follows from Eq. (27) that

$$
\begin{aligned}
\mathcal{W}_{\nu}\left(\hat{\boldsymbol{\phi}}_{1}, \hat{\boldsymbol{\phi}}_{2} ; z\right) & =\sum_{n} \kappa_{n} \mathcal{U}\left[\psi_{n}\left(\hat{\boldsymbol{\phi}}_{1} ; 0\right)\right] \mathcal{U}\left[\psi_{n}\left(\hat{\boldsymbol{\phi}}_{2} ; 0\right)\right]^{*} \\
& =\sum_{n} \kappa_{n} \psi_{n}\left(\hat{\boldsymbol{\phi}}_{1} ; z\right) \psi_{n}\left(\hat{\boldsymbol{\phi}}_{2} ; z\right)^{*}
\end{aligned}
$$

In other words, the cross-spectral density at the output plane can be found by separately transporting each 
coherent field using standard wave optics methods and forming the sum above.

If the fields $\{\psi\}$ form an orthonomal set, then we have "the" coherent mode expansion [20]. We distinguish the orthonormal coherent modes using $\Psi_{n}$, and it is straightforward to show that at $z=0$ these modes obey the following integral eigenvalue-type equation:

$$
\int d \hat{\boldsymbol{\phi}}_{2} \mathcal{W}_{\nu}\left(\hat{\boldsymbol{\phi}}_{1}, \hat{\boldsymbol{\phi}}_{2} ; 0\right) \Psi_{n}\left(\hat{\boldsymbol{\phi}}_{2} ; 0\right)=\kappa_{n} \Psi_{n}\left(\hat{\boldsymbol{\phi}}_{1} ; 0\right)
$$

In what follows we will present methods by which we can represent the undulator cross-spectral density as a coherent mode expansion (27) without solving the full problem (29). We begin in the limit that the electron beam emittance in both planes is much larger than the natural radiation emittance, $\varepsilon_{x, y} \gg \varepsilon_{r} \equiv \lambda / 4 \pi$. For this highly incoherent field there exist standard analytic solutions for both the cross-spectral density and the orthogonal coherent mode expansion [20]. Next, we consider the case typical to third-generation light sources, where only one emittance is large, $\varepsilon_{x} \gg \varepsilon_{r}$, while $\varepsilon_{y}$ is arbitrary. Under these conditions Ref. [19] showed that the 4D $\mathcal{W}_{\nu}$ can be divided into a product of the cross-spectral density in $x$ and $y$; the former again has an analytic expression, while the latter can be solved numerically with standard matrix eigensolvers. Finally, we discuss the general situation when no assumptions are made regarding the electron beam emittance. Rather than solving the full 4D eigenvalue problem, we show an alternative way to develop a coherent mode expansion that does not result in orthogonal modes. While this representation of $\mathcal{W}_{\nu}$ in terms of $\psi_{n}$ is not as compact, there are some situations in which it may be more computationally efficient than constructing an orthonormal set of modes. In any event, since our expansion is related to evaluating the $4 \mathrm{D}$ integration over the phase space coordinates, it will at least find use as an efficient means for determining $\mathcal{W}_{\nu}$ from which the problem (29) can be solved.

To be concrete, we will assume that the electron distribution function $f$ is Gaussian in both position and angle, and that the electron beam comes to a waist at the undulator middle:

$$
f\left(\hat{\boldsymbol{x}}, \hat{\boldsymbol{x}}^{\prime}\right)=\frac{\exp \left[-\frac{\hat{\hat{x}}^{2}}{\hat{\sigma}_{x}^{2}}-\frac{\hat{y}^{2}}{\hat{\sigma}_{y}^{2}}\right]}{\pi \hat{\sigma}_{x} \hat{\sigma}_{y}} \frac{\exp \left[-\frac{\hat{\hat{x}}^{2}}{\hat{\sigma}_{p_{x}}^{2}}-\frac{\hat{\hat{y}}^{2}}{\hat{\sigma}_{p_{y}}^{2}}\right]}{\pi \hat{\sigma}_{p_{x}} \hat{\sigma}_{p_{y}}},
$$

where the scaled rms beam sizes and divergences are $\hat{\sigma}_{x, y} \equiv \sigma_{x, y} / \sigma_{r}$ and $\hat{\sigma}_{p_{x, y}} \equiv \sigma_{p_{x, y}} / \sigma_{r^{\prime}}$, and there is no factor of $1 / 2$ in the exponential due to our definition $\hat{\boldsymbol{x}}=\sqrt{2} \boldsymbol{x} / \sigma_{r}$, etc. Then we can rewrite Eq. (21) as

$$
\begin{aligned}
\mathcal{W}_{\nu}\left(\hat{\boldsymbol{\phi}}_{1}, \hat{\boldsymbol{\phi}}_{2} ; 0\right)= & \frac{1}{2 \pi} e^{-\left[\hat{\sigma}_{x}^{2}\left(\hat{\phi}_{1}-\hat{\phi}_{2}\right)_{x}^{2}+\hat{\sigma}_{y}^{2}\left(\hat{\phi}_{1}-\hat{\phi}_{2}\right)_{y}^{2}\right] / 4} \\
& \times \int d \hat{\boldsymbol{x}}^{\prime} \frac{\exp \left[-\frac{x^{\prime 2}}{\hat{\sigma}_{p_{x}}^{2}}-\frac{y^{\prime 2}}{\hat{\sigma}_{P_{y}}^{2}}\right]}{\pi \hat{\sigma}_{x} \hat{\sigma}_{p_{x}}} \\
& \times \mathcal{E}_{\nu}^{0}\left(\hat{\boldsymbol{\phi}}_{1}-\hat{\boldsymbol{x}}^{\prime} ; 0\right) \mathcal{E}_{\nu}^{0}\left(\hat{\boldsymbol{\phi}}_{2}-\hat{\boldsymbol{x}}^{\prime} ; 0\right)^{*}
\end{aligned}
$$

\section{A. Incoherent emission: $\varepsilon_{x, y} \gg \lambda / 4 \pi$}

When the electron beam physical and angular widths are much larger than the natural size and divergence of the undulator field, $\sigma_{x} / \sigma_{r}=\hat{\sigma}_{x} \gg 1$ and $\sigma_{p_{x}} / \sigma_{r^{\prime}}=\hat{\sigma}_{p_{x}} \gg 1$, then the generated radiation is composed of many distinct coherent regions. The predominantly incoherent field can then be characterized transversely as having of order $\hat{\varepsilon}_{x} \hat{\varepsilon}_{y}=\left[\varepsilon_{x} /\left(\lambda_{r} / 4 \pi\right)\right]\left[\varepsilon_{y} /\left(\lambda_{r} / 4 \pi\right)\right] \gg 1$ independent modes. In this case many usual results of incoherent source theory may be applied including the van Cittert-Zernike theorem, and the cross-spectral density can be approximated using the Gaussian-Schell model. This incoherent limit has been studied extensively in the literature, and what we present here is mostly for review and to compare with the analysis in the next two sections.

Rather than use a more mathematical derivation of the incoherent limit as was done in, e.g., [19], we choose to base our results on physical arguments that are more in the spirit of Ref. [17]. The functional form of the ensemble averaged cross-spectral density is dominated by the particle phase space if $\varepsilon_{x, y} \gg \lambda / 4 \pi$, in which case the precise shape of the coherent undulator mode becomes relatively unimportant when calculating $\mathcal{W}_{\nu}$. Hence, we can approximate (31) by replacing the expression for $\mathcal{E}_{\nu}^{0}$ in (13) with any convenient single-peaked function that has the same flux and whose scaled width is of order unity. A simple choice that results in nice, closed-form analytic expressions uses a Gaussian approximation, with

$$
\mathcal{E}_{\nu}^{0, G}(\boldsymbol{\phi}) \propto e^{-\phi^{2} / 4 \sigma_{r^{\prime}}^{2}} \Rightarrow \mathcal{E}_{\nu}^{0, G}(\hat{\boldsymbol{\phi}})=\sqrt{\frac{2 \mathcal{F}_{\nu}}{\mathcal{F}_{1}}} e^{-\hat{\phi}^{2} / 2} .
$$

As alluded to earlier, the prefactor $\sqrt{2 \mathcal{F}_{\nu} / \mathcal{F}_{1}}$ insures that $\mathcal{E}_{\nu}^{0, G}$ has the correct flux.

Armed with the Gaussian field (32), we can now analytically compute an approximation to the cross-spectral density that is accurate provided $\hat{\sigma}_{x}, \hat{\sigma}_{y}, \hat{\sigma}_{p_{x}}, \hat{\sigma}_{p_{y}} \gg 1$; the resulting $\mathcal{W}_{\nu}$ is a separable function of $x$ and $y$, and in fact corresponds to the Gaussian-Schell model of undulator radiation. We have

$$
\begin{aligned}
\mathcal{W}_{\nu}\left(\hat{\boldsymbol{\phi}}_{1}, \hat{\boldsymbol{\phi}}_{2}\right) & \rightarrow \mathcal{W}_{\nu}^{\mathrm{GS}}\left(\hat{\boldsymbol{\phi}}_{1}, \hat{\boldsymbol{\phi}}_{2}\right) \\
& =\mathcal{W}_{\nu, x}^{\mathrm{GS}}\left(\hat{\boldsymbol{\phi}}_{1, x}, \hat{\boldsymbol{\phi}}_{2, x}\right) \mathcal{W}_{\nu, y}^{\mathrm{GS}}\left(\hat{\boldsymbol{\phi}}_{1, y}, \hat{\phi}_{2, y}\right),
\end{aligned}
$$

where the cross-spectral density along $x$ has the same functional form as that along $y$. Hence, we will only 
explicitly write out $\mathcal{W}_{\nu, x}^{\mathrm{GS}}$. To simplify the expression we define the scaled convolved width $\Sigma_{x}$ and divergence $\Sigma_{\phi_{x}}$; the former is given by the normalized e-beam width $\hat{\sigma}_{x} \equiv$ $\sigma_{x} / \sigma_{r}$ added in quadrature with the natural, unit radiation width, while the latter equals a similar sum of the normalized e-beam angular spread $\hat{\sigma}_{x^{\prime}} \equiv \sigma_{x^{\prime}} / \sigma_{r^{\prime}}$ and the scaled (unit) radiation divergence:

$$
\Sigma_{x, y} \equiv \sqrt{1+\hat{\sigma}_{x, y}^{2}}, \quad \Sigma_{\phi_{x, y}} \equiv \sqrt{1+\hat{\sigma}_{x^{\prime}, y^{\prime}}^{2}}
$$

In terms of these, the Gaussian-Schell cross-spectral density along $x$ is

$$
\begin{aligned}
\mathcal{W}_{\mathrm{GS}, x}= & \frac{\mathcal{F}_{\nu} / \mathcal{F}_{1}}{\sqrt{\pi} \Sigma_{\phi_{x}}} \exp \left[-\frac{\hat{\phi}_{1, x}^{2}+\hat{\phi}_{2, x}^{2}}{2 \Sigma_{\phi_{x}}^{2}}\right] \\
& \times \exp \left[-\frac{\hat{\Sigma}_{x}^{2} \hat{\Sigma}_{\phi_{x}}^{2}-1}{4 \hat{\Sigma}_{\phi_{x}}^{2}}\left(\hat{\phi}_{1, x}-\hat{\phi}_{2, x}\right)^{2}\right],
\end{aligned}
$$

with a similar expression in $y$. Note that while Eq. (35) makes mathematical sense for arbitrary electron beam parameters, the Gaussian approximation differs from the true $\mathcal{W}_{\nu}$ for undulator radiation in certain important ways as the beam emittance becomes small, i.e., as $\Sigma_{x}$ and/or $\Sigma_{\phi_{x}}$ approach unity. We will explore some of these differences in the following sections.

Before turning to the coherent mode expansion, we plot the Gaussian-Schell cross-correlation function $\tilde{\Gamma}_{\nu, y}=$ $\mathcal{W}_{\nu}\left(\phi_{1}+\xi_{x} / 2, \phi_{1}-\xi_{y} / 2\right)$ in Fig. 1 . Here we explicitly see that the rms width along physical angle $\phi_{x}=\left(\phi_{1}+\phi_{2}\right)_{x} / 2$ is $\sigma_{r^{\prime}} \Sigma_{y}=\sqrt{\sigma_{r^{\prime}}^{2}+\sigma_{x^{\prime}}^{2}}$, which also equals the rms width of the intensity in the angular representation. In addition, the correlation length in angle is directly proportional to the rms width along the difference coordinate $\left(\phi_{1}-\phi_{2}\right)_{x}$ which equals $2 \sigma_{r} / \Sigma_{x}=2 \sigma_{r} / \sqrt{\sigma_{r}^{2}+\sigma_{x}^{2}}$.

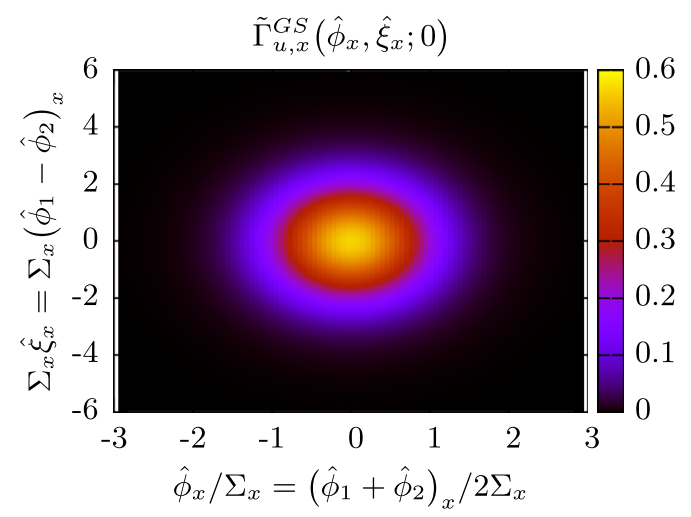

FIG. 1. Gaussian-Schell model cross-correlation function $\tilde{\Gamma}_{\nu, y}=\mathcal{W}_{\nu}\left(\phi_{1}+\xi_{x} / 2, \phi_{1}-\xi_{x} / 2\right)$ given by (35). The rms width in the average coordinate $\hat{\phi}_{x}$ is of order the total divergence $\Sigma_{\phi_{x}}$, while the correlation length along the difference coordinate $\hat{\xi}_{x}$ is $\sim 1 / \Sigma_{x}$
The orthogonal, coherent modes associated with the Gaussian-Schell model have convenient analytic expressions first derived in Ref. [29] (see also, e.g., [20,30]). If we write $\mathcal{W}_{\nu, x}^{\mathrm{GS}}=\sum_{n} \kappa_{n}^{\mathrm{GS}} \Psi_{n}^{\mathrm{GS}}$, the coherent modes $\Psi_{n}^{\mathrm{GS}}$ are Hermite-Gauss functions

$$
\begin{aligned}
\Psi_{n}^{\mathrm{GS}}\left(\hat{\phi}_{x}\right)= & \frac{\left(\Sigma_{\phi_{x}} / \Sigma_{x}\right)^{1 / 4}}{\sqrt{\sqrt{\pi} 2^{n} n !}} H_{n}\left(\sqrt{\frac{\Sigma_{\phi_{x}}}{\Sigma_{x}}} \hat{\phi}_{x}\right) \\
& \times \exp \left(-\frac{\Sigma_{\phi}}{2 \Sigma_{x}} \hat{\phi}_{x}^{2}\right),
\end{aligned}
$$

where $H_{n}(x)$ is the Hermite polynomial of order $n$, while the eigenvalue weight is

$$
\kappa_{n}^{\mathrm{GS}} \equiv \frac{2 \mathcal{F}_{\nu} / \mathcal{F}_{1}}{\Sigma_{\phi_{x}} \Sigma_{x}+1}\left(\frac{\Sigma_{\phi_{x}} \Sigma_{x}-1}{\Sigma_{\phi_{x}} \Sigma_{x}+1}\right)^{n} .
$$

The Gaussian-Schell model of undulator radiation has been discussed in many previous papers (see, e.g., [17,31]), while its coherent mode decomposition (36) and (37) was applied to study the propagation of $\mathrm{x}$ rays for both synchrotron light sources and FEL applications in Ref. [32]. Hence, we will only mention a few items. First, the orthogonal coherent mode representation permits simple expressions for the inverse number of modes $1 / M_{T}$ and the source strength $\mathscr{B}_{0}$ via

$$
\begin{aligned}
& \frac{1}{M_{T}}=\sum_{n} \kappa_{n}^{2}, \\
& \mathscr{B}_{0}=\sum_{n}(-1)^{p_{n}} \kappa_{n},
\end{aligned}
$$

where the parity $p_{n}$ is 0 if $\Psi_{n}$ is symmetric and +1 if $\Psi_{n}$ is antisymmetric. Since our normalization is $\sum_{n} \kappa_{n}=1$ with $\kappa_{n} \geq 0$, (38) clearly shows that $M_{T} \geq 1$ with equality holding if and only if there is only one mode and all other $\kappa_{n}$ vanish. While Eqs. (38) and (39) are true at $z=0$ and remain valid for propagation through free space and ideal lenses and mirrors, they do not apply after apertures or imperfect optical elements. This is because apertures, mirror slope errors, and the like typically involve transformations that do not preserve the orthogonality of the $\Psi_{n}$. Calculating $M_{T}$ when the $\Psi_{n}$ are not orthogonal requires computing the full $4 \mathrm{D}$ brightness function, so that in what follows we will for the most part use the simpler one to calculate $\mathscr{B}_{0}$ to characterize the source strength.

As an example of how one might use the coherent mode expansion (36) and (37) to transport the undulator field coherence, we consider the example of free-space propagation to an aperture, which is used in many $\mathrm{x}$-ray beam lines to improve the coherence. This simple situation was discussed at some length in terms of the coherent modes in Ref. [32], and is actually amenable to analytic 
TABLE I. Nominal source and e-beam parameters for undulator $\mathrm{A}$ at the Advanced Photon Source.

\begin{tabular}{lcc}
\hline \hline Parameter & Symbol & Value \\
\hline Wavelength & $\lambda_{r}$ & $1 \AA$ \\
Undulator length & $L_{u}$ & $2.31 \mathrm{~m}$ \\
Radiation rms size & $\sigma_{r}$ & $2.4 \mu \mathrm{m}$ \\
Radiation rms divergence & $\sigma_{r^{\prime}}$ & $3.3 \mu \mathrm{rad}$ \\
E-beam sizes & $\sigma_{x}$ & $70 \mu \mathrm{m}$ \\
& $\sigma_{y}$ & $8.4 \mu \mathrm{m}$ \\
E-beam emittances & $\varepsilon_{x}$ & $2.5 \mathrm{~nm}$ \\
& $\varepsilon_{y}$ & $0.025 \mathrm{~nm}$ \\
Scaled emittances & $\hat{\varepsilon}_{x}$ & 310 \\
& $\hat{\varepsilon}_{y}$ & 3.1 \\
\hline \hline
\end{tabular}

treatments. Nevertheless, we will characterize the improvement in coherence and loss of flux somewhat differently, in that we will show how both the flux $\mathcal{F}$ and the source strength $\mathscr{B}_{0}$ varies as one changes the slit widths $a_{x}$ and $a_{y}$ in both planes.

For this example we use parameters relevent to present operation at the Advanced Photon Source (APS) listed in Table I, which correspond to the nominal electron beam size and divergence for undulator A operating at $1 \AA$. Figure 2 plots the output scaled flux and source strength
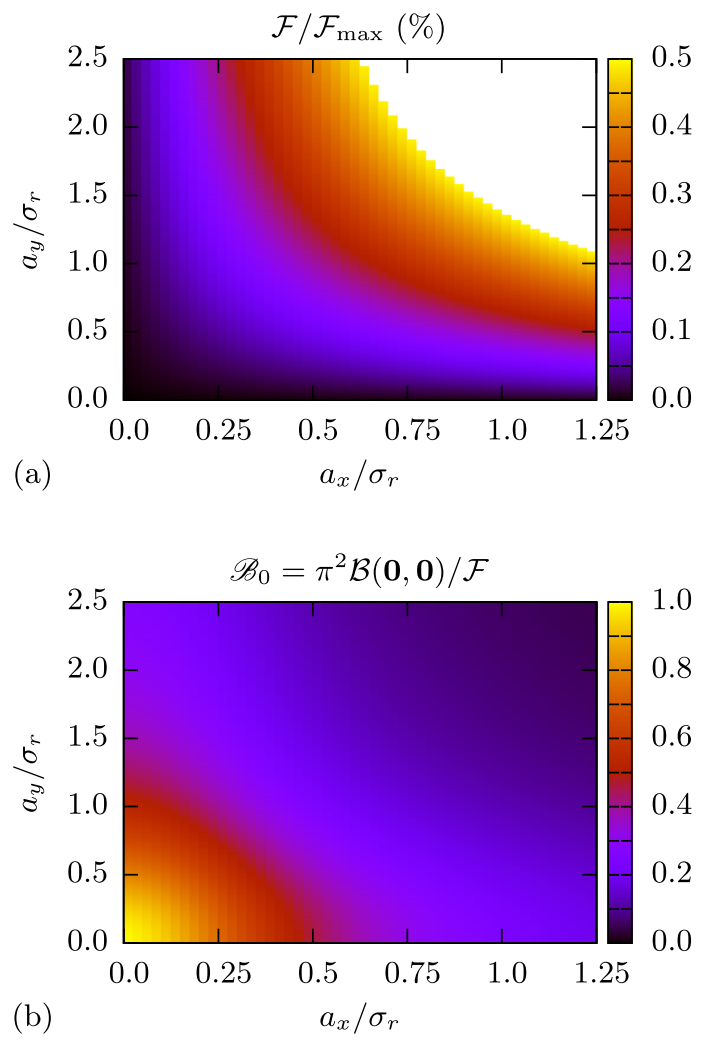

FIG. 2. Effect of a square slit of size $a_{x} \times a_{y}$ in the transverse plane on the Gaussian-Schell model of undulator radiation using the parameters in Table I. (a) plots how the flux decreases with slit size, while (b) gives the coherent fraction $\mathscr{B}_{0}$. To achieve $50 \%$ coherence one sacrifices nearly $99.9 \%$ of the flux since $1 /\left(\hat{\varepsilon}_{x} \hat{\varepsilon}_{y}\right) \sim 10^{-3}$. after being passed through an aperture that is located at $z=8 L_{u} \approx 18.5 \mathrm{~m}$ downstream and whose scaled widths in $x$ and $y$ are $a_{x} / \sigma_{r}$ and $a_{y} / \sigma_{r}$, respectively. To achieve significant coherence requires reducing the flux to $\sim 0.1 \%$ of its initial value, since $1 /\left(\hat{\varepsilon}_{x} \hat{\varepsilon}_{y}\right) \sim 10^{-3}$. Note that since the $y$ emittance is not large, $\hat{\varepsilon}_{y} \approx 3$, we expect that the Gaussian-Schell model used here will display some differences from the true undulator field. We will return to this example in the next section, where we will quantify the observed difference.

\section{B. Coherent mode expansion for third-generation sources: $\varepsilon_{x} \gg \lambda / 4 \pi$ with $\varepsilon_{y}$ arbitrary}

The equilibrium emittance in a storage ring results from a balance between the damping and diffusive effects of synchrotron emission. The natural horizontal emittance $\varepsilon_{x}$ is bounded from below according to the number and strength of bending magnets used to form a closed orbit, while in practice the equilibrium vertical emittance $\varepsilon_{y}$ is determined by the amount of coupling between the $x$ and $y$ directions. Modern third-generation storage rings typically operate with a vertical emittance $2-3$ orders of magnitude smaller than the horizontal emittance. As a result, there is a significant spectral range where $\varepsilon_{x} \gg \lambda / 4 \pi$ but $\varepsilon_{y}$ is of the order of the radiation emittance $\lambda / 4 \pi$, in which case we expect that the Gaussian-Schell model will not adequately capture all the physics. In this section we discuss the coherent mode expansion most relevant for thirdgeneration storage rings.

Reference [19] first derived an expression for the undulator radiation cross-spectral density in the limit that $\hat{\sigma}_{x}, \hat{\sigma}_{p_{x}} \gg 1$ but for arbitrary $\hat{\sigma}_{y}, \hat{\sigma}_{p_{y}}$. Their argument essentially went as follows: consider Eq. (31) for $\mathcal{W}_{\nu}$ under the substitution $\hat{x}^{\prime}=q+\left(\hat{\phi}_{1}+\hat{\phi}_{2}\right)_{x} / 2$ and $\hat{y}^{\prime}=p$,

$$
\begin{aligned}
\mathcal{W}_{\nu}= & \frac{1}{2 \pi} e^{-\left[\hat{\sigma}_{x}^{2}\left(\hat{\phi}_{1}-\hat{\phi}_{2}\right)_{x}^{2}+\hat{\sigma}_{y}^{2}\left(\hat{\phi}_{1}-\hat{\phi}_{2}\right)_{y}^{2}\right] / 4} \\
& \times \int d q d p \frac{\exp \left\{-\frac{\left[q+\left(\hat{\phi}_{1}+\hat{\phi}_{2}\right)_{x} / 2\right]^{2}}{\hat{\sigma}_{p_{x}}^{2}}-\frac{p^{2}}{\hat{\sigma}_{p_{y}}^{2}}\right\}}{\pi \hat{\sigma}_{x} \hat{\sigma}_{p_{x}}} \\
& \times \mathcal{E}_{\nu}^{0}\left(\frac{1}{2}\left(\hat{\phi}_{1}-\hat{\phi}_{2}\right)_{x}-q, \hat{\phi}_{1, y}-p\right) \\
& \times \mathcal{E}_{\nu}^{0}\left(\frac{1}{2}\left(\hat{\phi}_{2}-\hat{\phi}_{1}\right)_{x}-q, \hat{\phi}_{2, y}-p\right)^{*} .
\end{aligned}
$$

The exponential prefactor implies that $\mathcal{W}_{\nu}$ is negligible unless $\left(\hat{\phi}_{1}-\hat{\phi}_{2}\right)_{x}^{2} \lesssim 1 / \hat{\sigma}_{x}^{2} \ll 1$. Since the field $\mathcal{E}_{\nu}^{0}$ varies over a scale of order unity, we can therefore approximately set $\left(\hat{\phi}_{1}-\hat{\phi}_{2}\right)_{x}$ to zero in its argument. In addition, $\hat{\sigma}_{p_{x}}^{2} \gg 1$ means that the exponential in the integrand varies much more slowly than $\mathcal{E}_{\nu}^{0}$, so that we can set $q \rightarrow 0$ there.

Under these approximations the $x$ and $y$ dependencies factor, and in fact the cross-spectral density along $x$ can be 
recognized as the Gaussian-Schell model of Eq. (35) with the replacement $\left(\Sigma_{x}, \Sigma_{p_{x}}\right) \rightarrow\left(\hat{\sigma}_{x}, \hat{\sigma}_{p_{x}}\right)$. Hence, we find that

$$
\mathcal{W}_{\nu}\left(\hat{\boldsymbol{\phi}}_{1}, \hat{\boldsymbol{\phi}}_{2}\right) \rightarrow \mathcal{W}_{\nu, x}^{\mathrm{GS}}\left(\hat{\phi}_{1, y}, \hat{\phi}_{2, x}\right) \mathcal{W}_{\nu, y}\left(\hat{\phi}_{1, y}, \hat{\phi}_{2, y}\right)
$$

where the cross-spectral density along $y$ is

$$
\begin{aligned}
\mathcal{W}_{\nu, y}= & \frac{1}{2 \pi} \exp \left[-\frac{\hat{\sigma}_{y}^{2}}{4}\left(\hat{\phi}_{1, y}-\hat{\phi}_{2, y}\right)^{2}\right] \\
& \times \int d p d q \frac{e^{-p^{2} / \hat{\sigma}_{p_{y}}^{2}}}{\sqrt{\pi} \hat{\sigma}_{p_{y}}} \mathcal{E}_{\nu}^{0}\left(q, \hat{\phi}_{1, y}-p\right) \\
& \times \mathcal{E}_{\nu}^{0}\left(q, \hat{\phi}_{2, y}-p\right)^{*} .
\end{aligned}
$$

As mentioned previously, the undulator cross-spectral density for $\hat{\varepsilon}_{x} \gg 1$ and $\hat{\varepsilon}_{y}$ arbitrary was previously derived by Geloni et al. in Ref. [19]. Here, we present the first analysis of (42) in terms of its coherent modes.

The cross-spectral density $\mathcal{W}_{\nu, y}$ is two dimensional, meaning that we can approximate the eigenequation

$$
\int d \hat{\phi}_{1, y} \mathcal{W}_{\nu, y}\left(\hat{\phi}_{1, y}, \hat{\phi}_{2, y}\right) \Psi_{n}\left(\hat{\phi}_{1, y}\right)=\kappa_{n} \Psi_{n}\left(\hat{\phi}_{2, y}\right)
$$

with a discrete matrix equation that can be solved with a standard matrix eigensolver. Since $\mathcal{W}_{\nu, y}\left(\phi_{1}, \phi_{2}\right)$ at the source point is real and symmetric, we have used the easily implemented Jacobi eigenvalue algorithm to solve (43) with $\mathcal{W}_{\nu, y}\left(\phi_{1}, \phi_{2}\right)$ given by (42). Additionally, we choose to order the modes $\Psi_{n}$ such that the weights $\kappa_{n}$ are a monotonically decreasing function of $n$.

Given the more accurate but more computationally intensive expressions (42) and (43), it is natural to ask when we need them. Said another way, at what electron beam size and divergence is the Gaussian-Schell model sufficient to model undulator coherence? In Fig. 3 we graph three measures quantifying the difference in the crossspectral density between the valid for arbitrary $y$-emittance Eq. (42) and the Gaussian-Schell model (35) assuming that $\hat{\sigma}_{y}=\hat{\sigma}_{y^{\prime}}$. The red solid line plots the normalized difference in the inverse number of modes $1 / M_{T}$, while the green dashed line plots the computed difference of the source strength $\mathscr{B}_{0}$. The Gaussian-Schell model overestimates the level of coherence, thereby underestimating the number of modes. The difference is particularly pronounced at small but nonzero values of the emittance $\hat{\varepsilon}_{y}$ : the Gaussian-Schell model overestimates the degree of coherence by $\sim 40 \%$ when $\varepsilon_{y} \approx \lambda / 4 \pi$. The discrepancy in $1 / M_{T}$ is $35 \%$ and $13 \%$ when $\hat{\varepsilon}_{y}=0.25$ and $\hat{\varepsilon}_{y}=0$, respectively, while the same numbers for $\mathscr{B}_{0}$ are $27 \%$ and $0 \%$. The difference between the two model predictions decreases when $\hat{\varepsilon}_{y} \gtrsim 1$, and the agreement becomes better than $10 \%$ when $\hat{\varepsilon}_{y}>25$.

In addition, the blue dotted line in Fig. 3 plots the integrated rms difference in $\mathcal{W}_{\nu}$ defined via

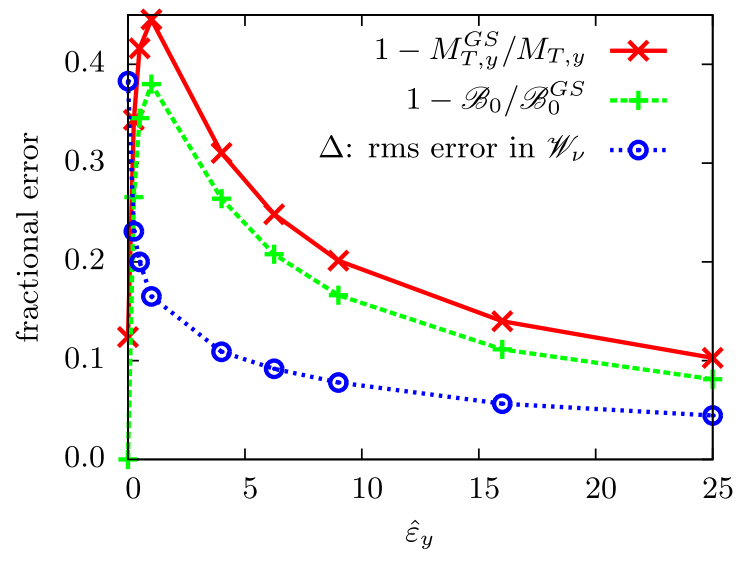

FIG. 3. Convergence of the mode representation toward the Gaussian-Schell model. The red solid line and green dashed line graph the normalized difference of two measures of coherence, the number of modes $M_{T}$ from Eq. (25) and the peak brightness divided by the flux $\mathscr{B}_{0}$ from Eq. (26). In both cases the GaussianSchell model underestimates the number of modes (overestimates the coherence). The blue dashed line quantifies the rms error $\Delta$ [Eq. (44)] when approximating $\mathcal{W}_{\nu, y}$ given by (42) with $\mathcal{W}_{\nu, y}^{\mathrm{GS}}$ from Eq. (35).

$$
\Delta^{2}=\frac{\int d \hat{\phi}_{1} d \hat{\phi}_{2}\left[\mathcal{W}_{\nu, y}\left(\hat{\phi}_{1}, \hat{\phi}_{2}\right)-\mathcal{W}_{\nu, y}^{\mathrm{GS}}\left(\hat{\phi}_{1}, \hat{\phi}_{2}\right)\right]^{2}}{\left[\int d \hat{\phi}_{1} d \hat{\phi}_{2} \mathcal{W}_{\nu, y}\left(\hat{\phi}_{1}, \hat{\phi}_{2}\right)\right]^{2}}
$$

In contrast to the coherence metrics $1 / M_{T}$ and $\mathscr{B}_{0}$, the difference $\Delta$ is a monotonically decreasing function of the emittance. As $\hat{\varepsilon}_{y}$ increases to 4 the rms difference quickly drops from $38 \%$ to $11 \%$, after which $\Delta$ decreases rather slowly. This behavior reflects the fact that the undulator coherence functions are highly non-Gaussian when the emittance is small. In terms of the brightness, the undulator $\mathcal{B}$ has negative regions when $\hat{\varepsilon}_{y}<1$ but is strictly positive for $\hat{\varepsilon}_{y} \geq 1$. Hence, when $\hat{\varepsilon}_{y} \geq 1$ we expect the field to look reasonably Gaussian, although with a different peak intensity and width as indicated by the coherence predictions of Fig. 3.

We show the degree to which the correlation function $\tilde{\Gamma}_{\nu, y}$ can differ from a Gaussian function in Fig. 4(a). Here, we plot the predicted $\tilde{\Gamma}_{\nu, y}$ in the limit $\hat{\varepsilon}_{y} \rightarrow 0$, which shows that there are significant oscillatory regions that are qualitatively different from the Gaussian-Schell model of Fig. 1. The central peak of the undulator field $\tilde{\Gamma}$ is also much more diamond shaped, although its widths along the two dimensions are of the same order as the GaussianSchell model.

It is also interesting to consider the spectral degree of coherence $\gamma_{\nu}$ as the vertical emittance approaches zero. We plot the far field $\left|\gamma_{\nu}\left(\hat{y}_{1}, \hat{y}_{2} ; z \rightarrow \infty\right)\right|$ in Fig. 4(b), whose functional form is given by $\tilde{\gamma}_{\nu}$ in the angular representation at $z=0$. Here, we see that the field is not completely coherent even as $\hat{\varepsilon}_{y} \rightarrow 0$. This fact was first described in 

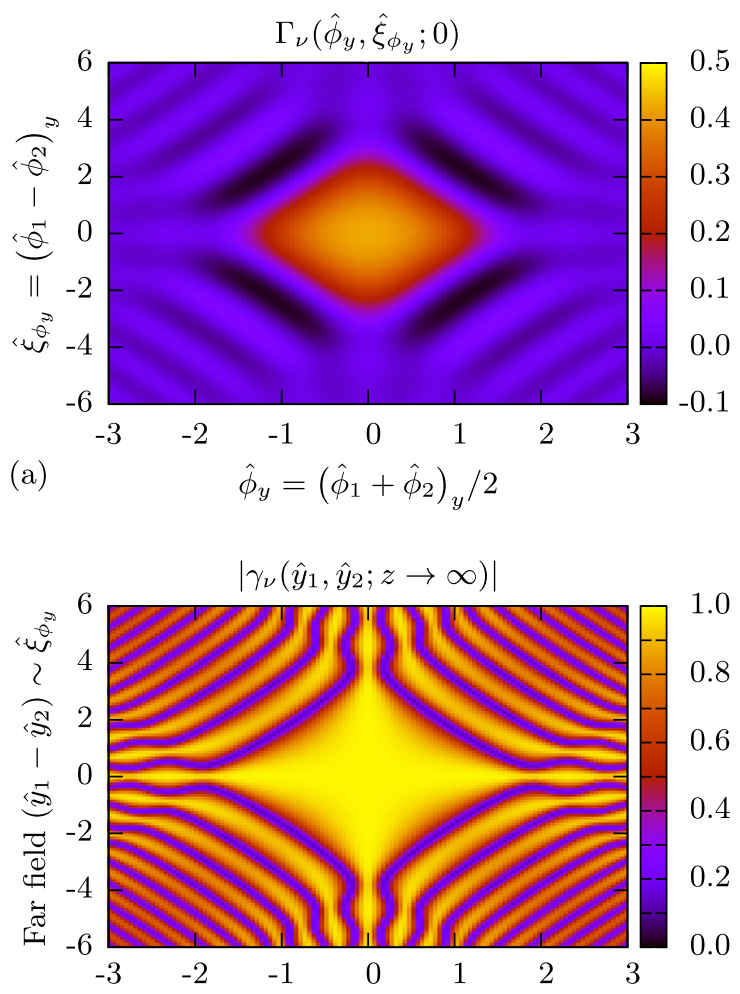

(b)

Far field $\left(\hat{y}_{1}+\hat{y}_{2}\right) \sim \hat{\phi}_{y}$

FIG. 4. Coherence functions in the limit $\hat{\varepsilon} \rightarrow 0$. Panel (a) plots $\tilde{\Gamma}_{\nu, y}=\mathcal{W}_{\nu}\left(\phi_{1}+\xi_{y} / 2, \phi_{1}-\xi_{y} / 2\right)$ from (42). Panel (b) plots the magnitude of the spectral degree of coherence $\left|\gamma_{\nu}\right|$ in the far field where $z \rightarrow \infty$. Since $\gamma_{\nu}$ in the far field is essentially the Fourier transform of the near field $\gamma_{\nu}$, (a) and (b) can be directly compared.

Ref. [19], where it was attributed to the fact that the undulator field $\mathcal{E}_{\nu}$ is not a separable function of $x^{\prime}$ and $y^{\prime}$, so that the large $\hat{\varepsilon}_{x}$ does have an effect on $\mathcal{W}_{\nu, y}$; mathematically this is evidenced by the integral over $q$ in Eq. (42).

While the lack of complete coherence can in principle be measured, we argue that it will be a rather small effect. In terms of the coherent modes, we find that the lowest order weight $\kappa_{0} \rightarrow 0.93$ as $\hat{\varepsilon}_{y} \rightarrow 0$, meaning that approximately 93\% of the flux is contained within the (non-Gaussian) lowest order mode $\Psi_{0}$. The modal purity along $y$ is $\approx 0.87 \%$, so that there are in some sense only $M_{T} \approx 1.15$ $y$ modes. This implies that the beam is largely coherent. In terms of the $\hat{y}_{1}-\hat{y}_{2}$ plane of Fig. 4(b), pinhole pair locations for which the degree of coherence $\left|\gamma_{\nu}\right| \approx 0$ also have a small intensity at one or both of the pinholes. In addition, we see that pinholes placed symmetrically about the optical axis lead to fringes of maximal visibility, and $\mathscr{B}_{0}=1$.

We can also investigate the undulator cross-spectral density $\mathcal{W}_{\nu, y}$ from Eq. (42) using its expansion into the coherent modes $\Psi_{n}$. For example, in Fig. 5(a) we plot the lowest order, fundamental mode $\Psi_{0}$ for four different
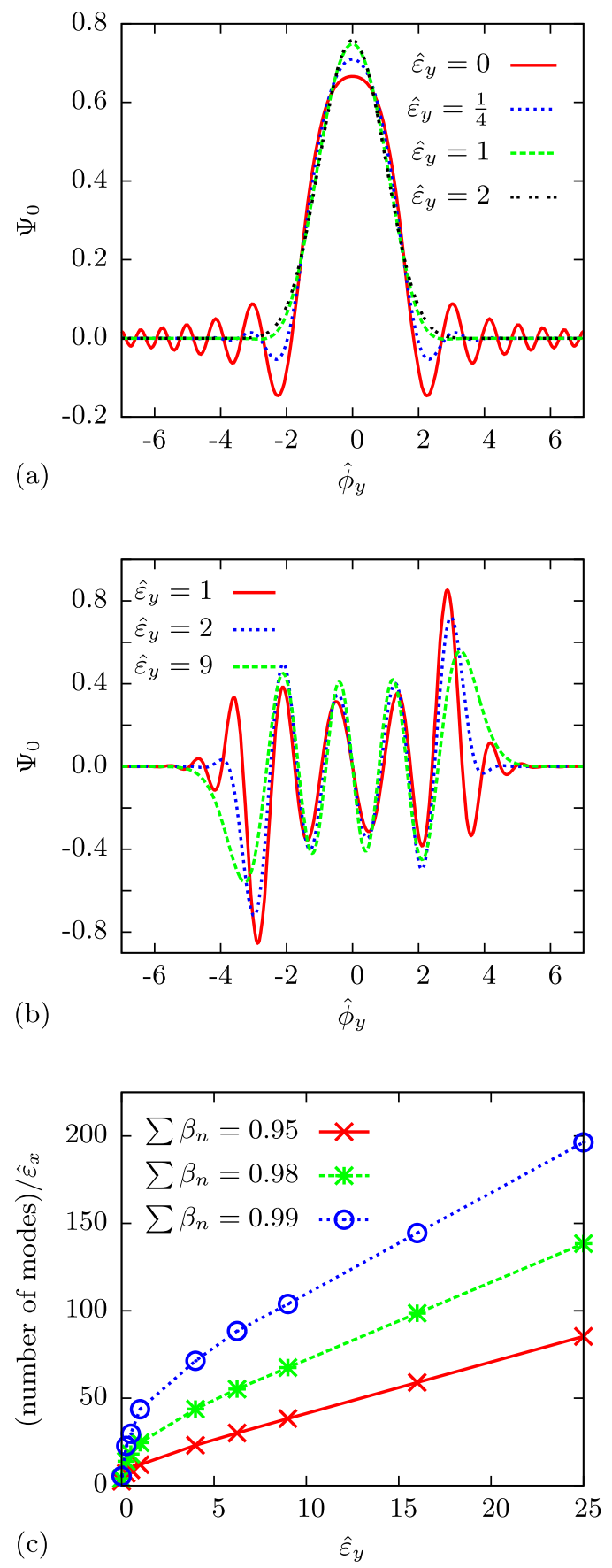

FIG. 5. Coherent mode expansion obtained by solving (42) and (43). (a) plots the lowest order mode $\Psi_{0}$ for four different values of the emittance. When $\varepsilon_{y} \rightarrow 0$ the mode is quite different than a Gaussian, but is nearly indistinguishable from the Gaussian prediction when $\hat{\varepsilon}_{y} \gtrsim 4$. (b) plots the eighth-order mode $\Psi_{7}$ for three different $y$ emittances. The Gaussian-Schell model would lie almost exactly on the line given by $\hat{\varepsilon}_{y}=9$. Panel (c) shows how the required number of coherent modes varies with the emittance $\hat{\varepsilon}_{y}$ for three different levels of total flux contained: the solid red, dashed green, and dotted blue lines have the minimum number of modes to contain $95 \%, 98 \%$, and $99 \%$ of the flux, respectively. 
values of the emittance $\hat{\varepsilon}_{y}$. The mode $\Psi_{0}$ exhibits slowly decaying, oscillatory tails when the emittance vanishes, as one might expect from the corresponding oscillations in the coherence function seen in Fig. 4(a). As the emittance increases the oscillatory part of $\Psi_{0}$ quickly becomes quite small, and when $\hat{\varepsilon}_{y} \gtrsim 4$ the lowest order mode is nearly indistinguishable from the Gaussian-Schell model $\Psi_{0}^{\mathrm{GS}}$. Panel (b) makes a similar comparison with the mode $\Psi_{7}$. When the $y$ emittance equals the radiation emittance, $\hat{\varepsilon}_{y}=1$, the solid red line of $\Psi_{7}$ shows significant qualitative differences from the corresponding Hermite-Gauss function; while the power in the mode is small $\left(\kappa_{7} \approx 7 \times 10^{-3}\right)$, it does need to be included if you want to capture more than 95\% of the flux. As the emittance increases the modes more closely resemble their Hermite-Gauss counterparts particularly at small to moderate $\tilde{\phi}_{y}$, as shown by the $\hat{\varepsilon}_{y}=2$ green dashed line; when $\hat{\varepsilon}_{y} \gtrsim 9$ the undulator $\Psi_{7}$ is essentially indistinguishable from its Gaussian-Schell counterpart.

Recalling that we have ordered the modes such that $\kappa_{n}$ decays with $n$, another natural question is "how many coherent modes are required to adequately represent the cross-spectral density $\mathcal{W}_{\nu}$ ?" We quantify this in Fig. 5(c), where we plot the number of modes per unit $\hat{\varepsilon}_{x}$ required to contain a certain fraction of the flux as a function the $\hat{\varepsilon}_{y}$. The red solid, green dashed, and blue dotted lines show how many modes are needed to capture $95 \%, 98 \%$, and 99\% of the flux, respectively (note that to get the total number of modes in $x$ and $y$ we must multiply by $\hat{\varepsilon}_{x} \gg 1$ ). We find that the number of modes grows quickly at small $\hat{\varepsilon}_{y} \lesssim 5$, after which it grows linearly with the $y$ emittance. It turns out that the slope of this linear part is the same as the Gaussian-Schell model at the same flux, although the Gaussian-Schell model consistently requires fewer modes to capture a given flux. In fact, if we were to include the corresponding Gaussian-Schell model lines in Fig. 5(c), the $95 \%, 98 \%$, and $99 \%$ flux lines would be parallel to those in Fig. 5(c), but the number of modes required for $\hat{\varepsilon}_{y}=25$ would be about 73,112 , and 145 per unit $\hat{\varepsilon}_{x}$, respectively, as compared to 86, 139, 197 from the figure. This indicates that the higher order modes associated with the undulator field (42) decay much more slowly than do their GaussianSchell counterparts, so that representing $\mathcal{W}_{\nu, y}$ to higher accuracy becomes an increasingly intensive computational proposition.

Finally, we conclude this section by reproducing Fig. 2 of the previous section, but this time we use the more accurate undulator model of $\mathcal{W}_{\nu, y}$ to compute the flux and brightness through the aperture. Again, we use the APS-style parameters at $1 \AA$ listed in Table I, and propagate the field $8 L_{u}$ downstream to a rectangular aperture with dimensions $a_{x} \times a_{y}$. We show the resulting flux $\mathcal{F}_{\nu}$ and source strength $\mathscr{B}_{0}$ in Figs. 6(a) and 6(b). The predicted reduction in flux and increase in coherence is qualitatively quite similar from what we predicted with the
Gaussian-Schell model in Figs. 2(a) and 2(b): at this propagation distance significant coherence requires a slit of width $\sim \sigma_{r}$ in each direction, in which case the flux is reduced to $\sim 1 /\left(\hat{\varepsilon}_{x} \hat{\varepsilon}_{y}\right) \sim 10^{-3}$ of its initial value.
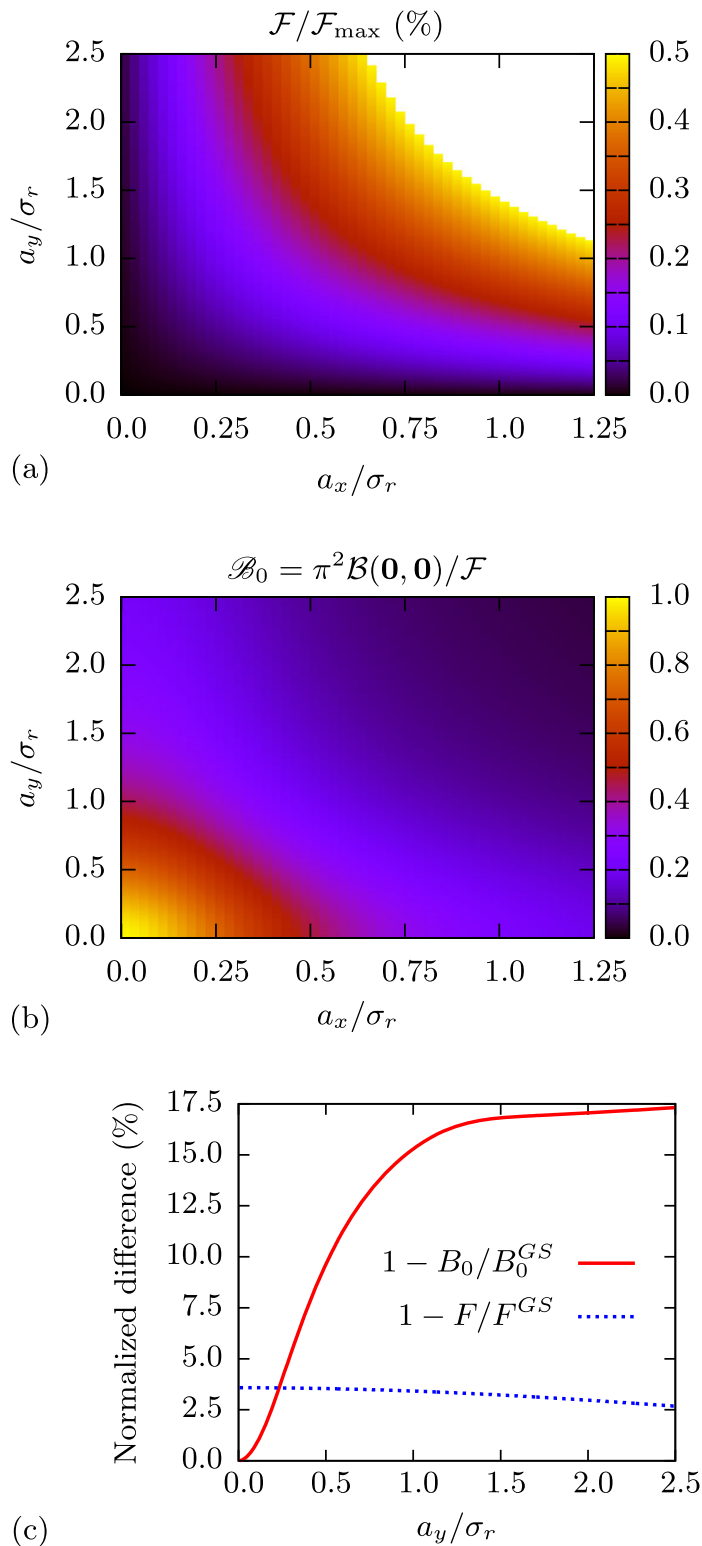

FIG. 6. Effect of a square slit of size $a_{x} \times a_{y}$ in the transverse plane on undulator radiation. The aperture is located downstream at $z=8 L_{u}$, and the undulator field is produced by a beam whose parameters are given in Table I. (a) plots how the flux decreases with slit size, while (b) gives the coherent fraction $\mathscr{B}_{0}$. To achieve a highly coherent beam with $\mathscr{B}_{0}>0.5$ requires a very small slit that sacrifices all but $\sim 1 /\left(\hat{\varepsilon}_{x} \hat{\varepsilon}_{y}\right) \sim 10^{-3}$ of the flux $\mathcal{F}$. Panel (c) compares the result here that decomposes the field using the more accurate (42) with the Gaussian-Schell model of the previous section. We see that the Gaussian-Schell model overestimates the flux and source strength, but that the agreement is reasonably good for the very small apertures required to produce highly coherent $\mathrm{x}$ rays. 
In Fig. 6(c) we plot the difference in the source strength $\mathscr{B}_{0}$ and the total flux $\mathcal{F}$ between the undulator model shown here and the Gaussian-Schell result from the previous section. Here, the discrepancy depends only on the slit width in $y$, since both models use the GaussianSchell approximation along $x$ appropriate for $\hat{\varepsilon}_{x} \gg 1$. Figure 6(c) indicates that the Gaussian-Schell model approximates the flux through the slit to better than $4 \%$, while the accuracy of the predicted source strength depends on the slit width; for small slits and high degrees of $y$ coherence the Gaussian-Schell model is quite accurate, while when $a_{y}>\sigma_{r}$ and $\mathscr{B}_{0}<0.5$ it overestimates the source strength by $15 \%-18 \%$.

\section{A coherent mode representation for arbitrary electron beam emittance}

In the previous two sections we have discussed the coherent mode expansion of undulator radiation when at least one of the e-beam emittances is large, $\varepsilon_{x} \gg \lambda / 4 \pi$. While this limit typically applies to the current generation of storage rings, future plans call for light sources whose emittance satisfies $\varepsilon_{x, y} \lesssim \lambda / 4 \pi$ over a significant x-ray spectral range. So-called diffraction-limited storage rings (or, more colloquially, "ultimate" storage rings) based on multibend achromats (MBAs) are currently in the construction or design phase at several locations around the globe, with the hopes of opening up entirely new avenues of coherent $\mathrm{x}$-ray imaging. In this section we discuss a coherent mode representation applicable for arbitrary $x-y$ emittances.

The previous discussion relied on the fact that when $\hat{\varepsilon}_{x} \gg 1$ the cross-spectral density $\mathcal{W}_{\nu}$ becomes a separable function of $\phi_{x}$ and $\phi_{y}$. In this case the resulting $\mathcal{W}_{\nu, x}$ was describable in terms of the Gaussian-Schell model, while $\mathcal{W}_{\nu, y}$ was either also Gaussian-Schell if $\hat{\varepsilon}_{y} \gg 1$, or more generally could be decomposed numerically into coherent modes using a standard matrix eigensolver.

When the emittance in both planes is small, however, the coherent modes are nonseparable functions of both $\phi_{x}$ and $\phi_{y}$. Hence, obtaining the 2D orthogonal coherent modes requires solving the eigenequation (29) of the 4D field $\mathcal{W}_{\nu}$. We have decided that such a procedure is beyond the scope of this paper, and instead we look for another coherent mode representation that can be constructed without ever having to directly deal with a 4D field. The decomposition we present is essentially related to numerically integrating the expression for the cross-spectral density Eq. (21). Hence, some of our analysis will be applicable to the full coherent mode eigenproblem, as solving (29) requires first determining $\mathcal{W}_{\nu}$ from either (21) or (31).

To begin our discussion, consider the numerical quadrature

$$
\int d y g\left(x_{1}, y\right) g\left(x_{2}, y\right)^{*} \approx \sum_{n} w_{n} g\left(x_{1}, y_{n}\right) g\left(x_{2}, y_{n}\right)^{*},
$$

where the weights $w_{n}$ and points $x_{n}$ depend on the integration scheme, and the number of terms in the sum is chosen to give reasonable accuracy. Applying this to the cross-spectral density (21), we write

$$
\begin{aligned}
& \mathcal{W}_{\nu}\left(\hat{\boldsymbol{\phi}}_{1}, \hat{\boldsymbol{\phi}}_{2} ; 0\right) \\
& \approx \sum_{n} w_{n} \frac{f\left(\hat{\boldsymbol{x}}_{n}, \hat{\boldsymbol{x}}_{n}^{\prime}\right)}{2 \pi}\left[e^{-i \hat{\phi}_{1} \cdot \hat{\boldsymbol{x}}_{n}} \mathcal{E}_{\nu}^{0}\left(\hat{\boldsymbol{\phi}}_{1}-\hat{\boldsymbol{x}}_{n}^{\prime} ; 0\right)\right] \\
& \quad \times\left[e^{-i \hat{\boldsymbol{\phi}}_{2} \cdot \hat{\boldsymbol{x}}_{n}} \mathcal{E}_{\nu}^{0}\left(\hat{\boldsymbol{\phi}}_{2}-\hat{\boldsymbol{x}}_{n}^{\prime} ; 0\right)\right] *
\end{aligned}
$$

Equation (46) is a coherent mode expansion of the form given by Eq. (27) if we identify $\psi_{n}$ with the quantity in square brackets,

$$
\psi_{n}\left(\hat{\boldsymbol{\phi}}_{1} ; 0\right)=e^{-i \hat{\boldsymbol{\phi}}_{1} \cdot \hat{\boldsymbol{x}}_{n}} \mathcal{E}_{\nu}^{0}\left(\hat{\boldsymbol{\phi}}_{1}-\hat{\boldsymbol{x}}_{n}^{\prime} ; 0\right),
$$

while the mode expansion coefficient is proportional to the product of the distribution function and the quadrature weight, $\kappa_{n}=w_{n} f\left(\hat{\boldsymbol{x}}_{n}, \hat{\boldsymbol{x}}_{n}^{\prime}\right) / 2 \pi$. We must stress that while this representation looks similar to those of the previous two sections, the modes $\psi_{n}$ need not be orthogonal and hence the representation (46) will typically require more terms. Nevertheless, since our discussion regarding the propagation of $\mathcal{W}_{\nu}$ via $\psi_{n}$ did not require the modes to be orthogonal, Eq. (46) is a completely valid coherent mode representation suitable for our purposes. In particular, as we showed with the propagation result (28), the cross-spectral density at the end of an optical transport line can be found by propagating the coherent modes (47) through the beam line elements and then forming the sum

$$
\mathcal{W}_{\nu}\left(\hat{\boldsymbol{\phi}}_{1}, \hat{\boldsymbol{\phi}}_{2} ; z\right)=\sum_{n} w_{n} \psi_{n}\left(\hat{\boldsymbol{\phi}}_{1} ; z\right) \psi_{n}\left(\hat{\boldsymbol{\phi}}_{2} ; z\right)^{*}
$$

at the output plane. Hence, the second-order coherence anywhere along the transport line is known once we choose an appropriate set of coordinates $\left(\hat{\boldsymbol{x}}_{n}, \hat{\boldsymbol{x}}_{n}^{\prime}\right)$ and corresponding weights $w_{n}$.

There are many choices of $\left(\hat{\boldsymbol{x}}_{n}, \hat{\boldsymbol{x}}_{n}^{\prime}, w_{n}\right)$ that will encode the cross-spectral density as in (48), and our goal is to find the most efficient such representation. Since $\mathcal{W}_{\nu}$ is determined at the source, this task is equivalent to finding the most efficient way to compute Eq. (46). In other words, what numerical integration scheme leads to an acceptable level of accuracy with the smallest number of points? We began by trying several standard quadrature rules, including the usual Gaussian quadrature, a modified Gaussian quadrature in which the distribution $f$ is expanded in terms of Hermite functions, Clenshaw-Curtis quadrature, and several Newton-Cotes methods. Of these methods, we found that the most accurate and compact representation of (46) used the simple trapezoidal rule. The basic reason for this is that integrating an analytic function by the composite trapezoidal rule results in two types of errors: the 
first is a discretization error that decreases exponentially with the number of points, while the second is a boundary error that is proportional to the discontinuity of the function (or its derivatives) at the endpoints. Hence, numerically integrating periodic, analytic functions with the trapezoidal rule enjoys exponential convergence with the number of points. In our case, the integrand is analytic but not periodic, but the error terms at the boundary can be made exponentially small since $f$ in the integrand involves a Gaussian function (for much more on this topic see the recent review [33] and references therein).

Nevertheless, using the composite trapezoidal rule to define the coherent mode representation is very inefficient. In fact, we found that getting reasonable accuracy required $\sim 50$ modes for every $\lambda / 4 \pi$ units of emittance in both $x$ and $y$, beyond which the convergence was quite rapid. In other words, describing the radiation produced by an e-beam with scaled emittances $\hat{\varepsilon}_{x, y}=\varepsilon_{x, y} /(\lambda / 4 \pi)$ requires a total of $\sim\left(50 \hat{\varepsilon}_{x}\right)\left(50 \hat{\varepsilon}_{y}\right)=2500 \hat{\varepsilon}_{x} \hat{\varepsilon}_{y}$ modes; for comparison, the orthogonal mode decompositions discussed previously would need $\sim 400$ times fewer modes.

It is perhaps not surprising that usual quadrature schemes require so many points for the 4D integral (21), since such deterministic methods have computational requirements that scale exponentially with the number of dimensions. To try and circumvent this "curse of dimensionality," next we tried applying Monte Carlo integration techniques to evaluate $\mathcal{W}_{\nu}$. Since the integral already contains the electron probability distribution function $f$, the crossspectral density is most naturally computed using the importance sampling method of Monte Carlo integration (see, e.g., [34]). In this case, an estimate of the expected value is given by the sum

$$
\begin{aligned}
& \mathcal{W}_{\nu}\left(\hat{\boldsymbol{\phi}}_{1}, \hat{\boldsymbol{\phi}}_{2} ; 0\right) \\
& \approx \frac{1}{2 \pi N} \sum_{n=1}^{N}\left[e^{-i \hat{\boldsymbol{\phi}}_{1} \cdot \hat{\boldsymbol{x}}_{n}} \mathcal{E}_{\nu}^{0}\left(\hat{\boldsymbol{\phi}}_{1}-\hat{\boldsymbol{x}}_{n}^{\prime} ; 0\right)\right] \\
& \quad \times\left[e^{-i \hat{\boldsymbol{\phi}}_{2} \cdot \hat{x}_{n}} \mathcal{E}_{\nu}^{0}\left(\hat{\boldsymbol{\phi}}_{2}-\hat{\boldsymbol{x}}_{n}^{\prime} ; 0\right)\right]^{*},
\end{aligned}
$$

where the electron phase space coordinates $\left(\hat{\boldsymbol{x}}_{n}, \hat{\boldsymbol{x}}_{n}^{\prime}\right)$ are Gaussian distributed according to $f$.

We can think of (49) as an approximation of $\mathcal{W}_{\nu}$ that is constructed from the sum over the cross-spectral densities produced by a representative population of $N$ electrons, with $N \ll N_{e}$. The representation (49) will in general converge to the cross-spectral density $\mathcal{W}_{\nu}$ as we increase the number of sampled electrons $N$, but the rate of convergence will depend on how we select the coordinates $\hat{\boldsymbol{x}}_{n}$ and $\hat{\boldsymbol{x}}_{n}^{\prime}$.

Perhaps the simplest way to construct the electron population employs Gaussian random numbers to generate the particle positions and angles. While this random sampling Monte Carlo method is vastly superior to the composite trapezoidal rule mentioned earlier, it can be further improved by more uniformly sampling the electron phase space.

The reason that random sampling is not optimal is because it tends to be too "clumpy," exhibiting relative fluctuation levels $\sim 1 / \sqrt{N}$ that are much larger than those of the actual system. Similar difficulties arise in any simulation that models a many-body system with a relatively small number of particles, and the basic solution is to try to find a way to more uniformly distribute the sample coordinates. We can meet this objective by replacing the random samples for $\left(\hat{\boldsymbol{x}}_{n}, \hat{\boldsymbol{x}}_{n}^{\prime}\right)$ with four low discrepancy Halton sequences [35]. In short, Halton sequences are a family of deterministic sequences that fill the unit interval more uniformly for a given finite $N$ than do random sequences. Each Halton sequence is constructed by subdividing the unit interval by successive powers of a prime base, so that the first four prime numbers can be used for the $4 \mathrm{D}$ phase space.

Since the Halton sequence uniformly spans the unit interval, we can use the standard Box-Muller transform (without rejection sampling) [36] to convert these points into Gaussian distributed numbers appropriate for the phase space coordinates. For example, we can smoothly fill the 2D phase space $\left(\hat{x}_{n}, \hat{x}_{n}^{\prime}\right)$ with a random-looking set of Gaussian distributed points by pairing the transformed Halton sequence of base 2 with that of base 3 .

Figure 7 compares the performance of using Monte Carlo integration with randomly distributed points in panel (a) with that using the low-discrepancy Halton sequence in panel (b). Here, both graphs plot the measured error as a function of the number of coherent modes simulated per unit scaled emittance product $\hat{\varepsilon}_{x} \hat{\varepsilon}_{y}$. To make this plot, we chose a variety of emittances such that $1 \leq \hat{\varepsilon}_{x, y} \leq 24$, and each point is the average of 25 separate trials using either a distinct random seed or a different portion of the Halton sequence.

The first thing that should be evident from Fig. 7(a) is that the Monte Carlo sampling method is far more efficient at determining $\mathcal{W}_{\nu}$ than are classical numerical integration techniques: a 5\% accuracy is achieved here with more than an order of magnitude fewer fields than that required by the trapezoidal method. In addition, the error in source strength $\mathscr{B}_{0}$ scales roughly as $1 / \sqrt{N}$ (as we might naively guess), while the rms error $\Delta$ converges somewhat more slowly.

Although the usual random Monte Carlo sampling performs rather well, the quasirandom Monte Carlo algorithm employing low-discrepancy sequences does almost a factor of 10 better. For the same 5\% level of error, this method only requires about 15 modes per scaled emittance product $\hat{\varepsilon}_{x} \hat{\varepsilon}_{y}$. This number is beginning to be competitive with the orthogonal mode expansions of the previous sections, being only a factor of 4-5 more modes.

Now that we can construct the cross-spectral density for arbitrary emittance, we can investigate its properties. While in this paper we only look at the cross-spectral density at 

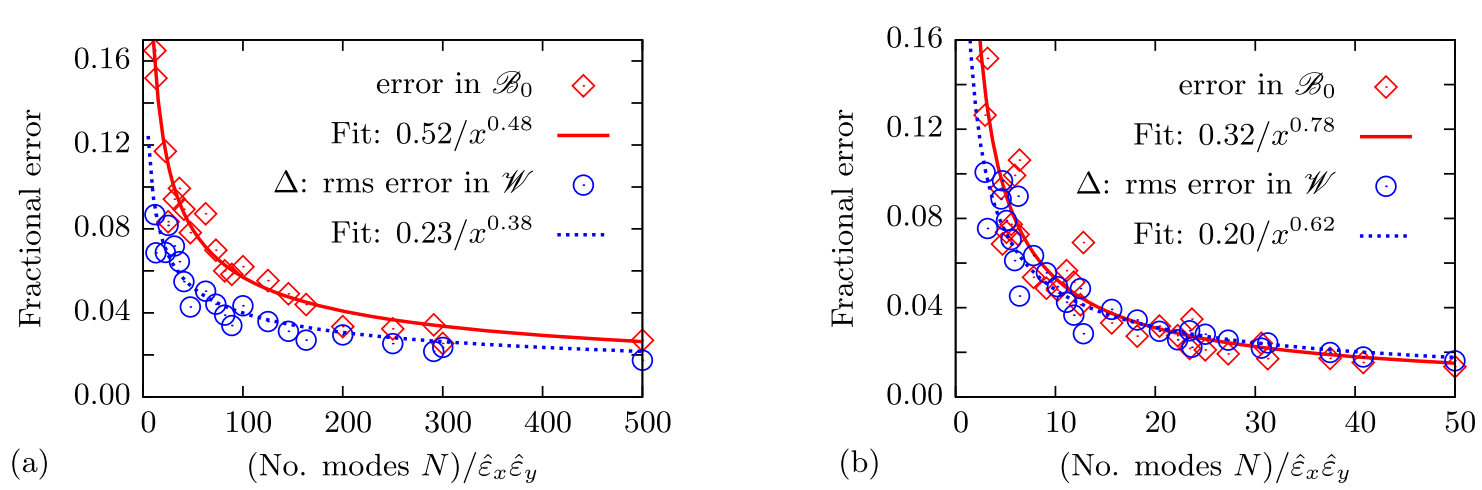

FIG. 7. Dependence of two measures of the error in the coherence function as a function of the number of simulated modes per unit phase space area. As shown in (a), distributing the electrons randomly results in rather slow convergence, requiring $\sim 150$ modes per transverse area $\hat{\varepsilon}_{x} \hat{\varepsilon}_{y}$ to achieve $\lesssim 5 \%$ accuracy. On the other hand, panel (b) demonstrates that employing a low-discrepancy sequence to distribute the electrons significantly reduces the required number of electrons; here, about 15 modes per $\hat{\varepsilon}_{x} \hat{\varepsilon}_{y}$ are sufficient to attain an error of $\lesssim 5 \%$. This number is a little more than 4 times the number of modes required for the orthogonal expansion valid when $\hat{\varepsilon}_{x} \gg 1$, and about 5 times more than the Gaussian-Schell model prediction.

the source, it is important to recognize that $\mathcal{W}_{\nu}$ at any point down the beam line can be constructed by propagating the coherent fields $\psi_{n}$ and summing as in (48). We will consider parameters that can be achieved with advanced diffraction-limited storage rings based on MBAs, for which $\varepsilon_{y} \leq \varepsilon_{x} \lesssim \lambda$. As mentioned earlier, in a storage ring the emittance along $x$ is dictated by the balance between damping and diffusion provided by the stochastic emission of synchrotron radiation, while $\varepsilon_{y}$ is set by the coupling. In particular, if the damping along $x$ and $y$ are the same, the emittances are given by

$$
\varepsilon_{x}=\frac{1}{1+r} \varepsilon_{0}, \quad \varepsilon_{y}=\frac{r}{1+r} \varepsilon_{0},
$$

where the natural emittance $\varepsilon_{0}$ is determined by the magnetic lattice, and the emittance ratio $r \equiv \varepsilon_{y} / \varepsilon_{x}$ satisfies $0 \leq r \leq 1$.

In diffraction-limited storage rings the natural emittance $\varepsilon_{0}$ is small enough that one can consider nearly round beams; in fact, to reduce Coulomb scattering that can both degrade the emittance and reduce the lifetime, the typical operating point of an MBA has $r \gtrsim 0.1$. In Fig. 8(a) we show how changing the electron beam focusing affects the x-ray coherence for such a ring, plotting the coherence metric $\mathscr{B}_{0}$ as a function of the electron beam $\beta_{x}=\sigma_{x}^{2} / \varepsilon_{x}$ at the undulator middle, assuming $\beta_{y}=L_{u} / \pi$. Here, we also include several different values of the emittance ratio assuming that the natural emittance $\varepsilon_{0}=\lambda / 2 \pi$ (i.e., $\hat{\varepsilon}_{0}=2$ ).

Figure 8(a) shows that the coherence is maximized when $\beta_{x} \approx L_{u} / \pi$ (or $\hat{\beta}_{x} \approx 1$ ), which indicates that the "natural" Rayleigh range of undulator radiation $Z_{R} \approx \beta_{x} \approx L_{u} / \pi$. Unfortunately, it is nearly impossible for lattice designers to make the beta functions in both $x$ and $y$ be simultaneously that small, and typically $\beta_{x}>3 L_{u} / \pi$.
Finally, we conclude this section by showing a few examples of the dominant coherent eigenmode $\Psi_{0}$, i.e., the orthogonal mode with highest weight $\kappa_{0}$. We extract the dominant coherent eigenmode using power iteration. Power iteration gives successive approximations to the dominant mode; for our particular application the $(k+1)$ th iteration is related to the $k$ th approximation by

$$
\Psi_{0}^{k+1}(\hat{\boldsymbol{\phi}})=\frac{\int d \hat{\boldsymbol{\phi}}^{\prime} \mathcal{W}\left(\hat{\boldsymbol{\phi}}, \hat{\boldsymbol{\phi}}^{\prime}\right) \Psi_{0}^{k}\left(\hat{\boldsymbol{\phi}}^{\prime}\right)}{\left[\int d \hat{\boldsymbol{\phi}} d \hat{\boldsymbol{\phi}}^{\prime} \mathcal{W}\left(\hat{\boldsymbol{\phi}}, \hat{\boldsymbol{\phi}}^{\prime}\right) \Psi_{0}^{k}\left(\hat{\boldsymbol{\phi}}^{\prime}\right)\right]^{1 / 2}},
$$

and the first guess $\Psi_{0}^{k=0}$ can be chosen to be the field produced by a single electron, Eq. (13). The power iteration method converges very quickly to the lowest order mode, typically requiring of order 5-10 iterations to obtain a reasonably accurate result when the undulator field (13) is used for the first guess $\Psi_{0}^{k=0}$. One way to test the convergence is to track the change in rms value of the field after successive iterations. In the cases we have checked the integrated rms error drops below the $1 \%$ level after a few iterations, and differences in successive approximations become $<10^{-4}$ after 5-6 iterations.

We plot profiles along $y$ and $x$ of dominant eigenmode in Figs. 8(b) and 8(c), where again we take $\varepsilon_{0}=\lambda / 2 \pi$. In the limit $\varepsilon_{y} \rightarrow 0$ the profile along $y$ approaches that produced by a single electron, while $\Psi_{0}\left(\phi_{y}\right)$ is reasonably well approximated by a Gaussian $\propto \exp -\hat{\phi}_{y}^{2} / 2$ when $\varepsilon_{y}=\varepsilon_{x}=\lambda / 4 \pi$. We note that at different values of the electron beam focusing $\beta_{x}$ these profiles are qualitatively very similar: the profiles when $\beta_{x}$ is increased by a factor of 16 can be approximately obtained by multiplying those in Fig. 8(b) by 1.3. Figure 8(c) plots the profiles along $x$ as we vary $\beta_{x}$. Each plot is approximately Gaussian, and we see that the angular spread decreases as $\beta_{x}$ increases. More careful inspection shows that the width of the angular 

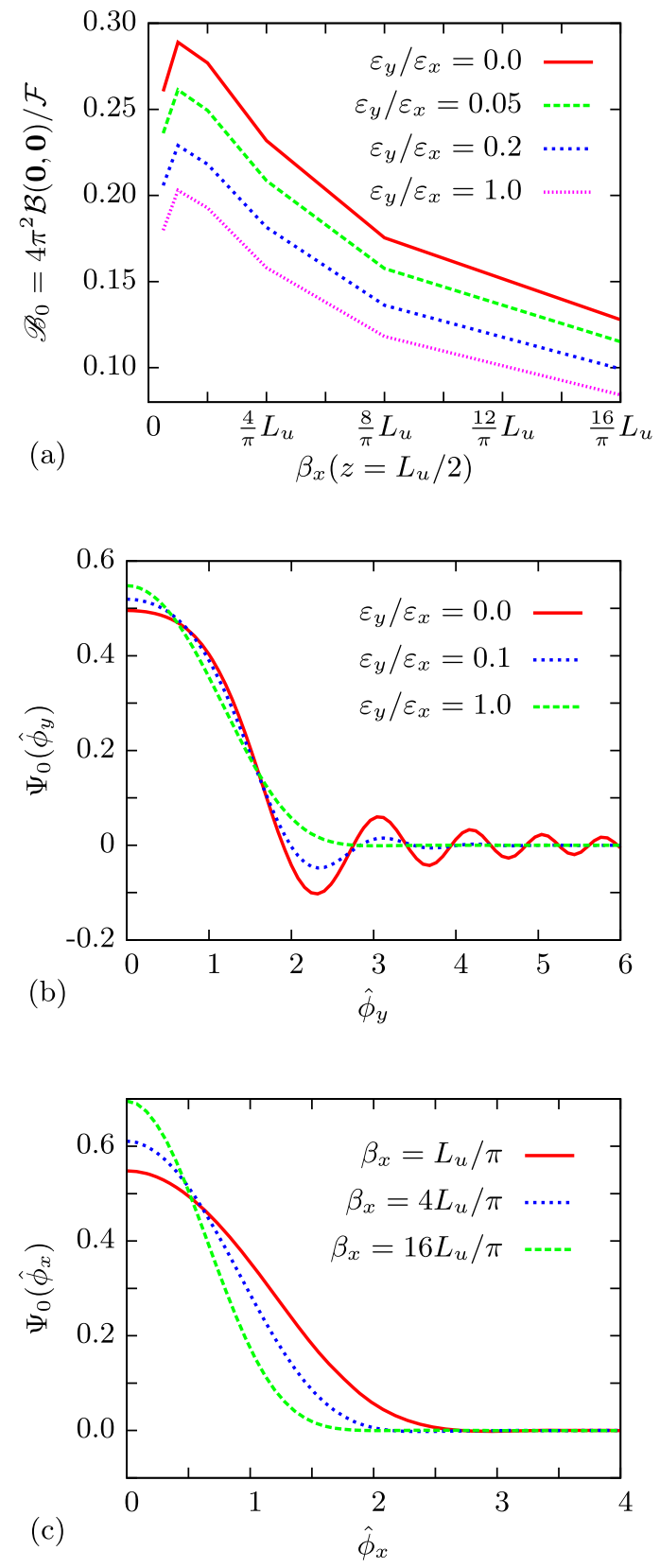

FIG. 8. (a) Coherence metric $\mathscr{B}_{0}$ as a function of the e-beam focusing for various emittance ratios. (b) Field profile along $y$ of the lowest order coherent eigenmode for different emittance ratios when $\beta_{x}=L_{u} / \pi . \Psi_{0}\left(\phi_{y}\right)$ is qualitatively the same at different $\beta_{x}$. (c) $\Psi_{0}\left(\phi_{x}\right)$ for various e-beam focusing when $\varepsilon_{y}=0$; this profile is nearly independent of the emittance ratio when $\varepsilon_{0}=\lambda / 2 \pi$.

intensity scales as $1 / \sqrt{\beta_{x}}$, which in turn implies that the Rayleigh range of the dominant coherent eigenmode is directly proportional to $\beta_{x}$. Hence, we conclude that the focusing parameters of $\Psi_{0}$ roughly match those of the electron beam when the emittance $\varepsilon_{x} \gtrsim \lambda / 4 \pi$. For smaller emittances similar scalings may apply, but in this case the dominant coherent mode is not Gaussian-like.

\section{CONCLUSIONS}

In this paper we have described three different coherent mode representations of partially coherent undulator radiation. We began with the well-known Gaussian-Schell decomposition in terms of Gauss-Hermite modes, which is valid provided the electron beam emittance is much larger than the natural radiation emittance $\lambda / 4 \pi$. In this largely incoherent case the specifics of the single-electron undulator field are unimportant. We then refined our analysis to include the situation when the electron beam emittance $\varepsilon_{y}$ in one direction is arbitrary, and found that the modes along $y$ are determined by solving a matrix eigenproblem. These coherent mode profiles can differ in important ways from the Gaussian-Schell case, particularly when $\varepsilon_{y}<\lambda / 4 \pi$ or when one considers higher order modes. Finally, we found that when the emittance in both planes is small, the mode profiles are inherently 2D. Rather than solve the associated 4D eigenproblem, we showed how to construct a set of coherent modes using a Monte Carlo approach that samples the electron phase space using low-discrepancy sequences. While not as elegant as the other methods, it still provides a rather compact representation of the partially coherent $\mathrm{x}$ rays suitable for propagation using standard wave optics codes.

\section{ACKNOWLEDGMENTS}

We would like to thank our colleagues Ruben Reininger, Xianbo Shi, and Lahsen Asoufid from the X-ray Sciences Division for useful discussions. This work was supported by the U.S. Department of Energy Office of Science under Contract No. DE-AC02-06CH11357.

[1] M. Eriksson, J.F. van der Veen, and C. Quitmann, J. Synchrotron Radiat. 21, 837 (2014).

[2] R. Hettel, J. Synchrotron Radiat. 21, 843 (2014).

[3] D. Einfeld, M. Plesko, and J. Schaper, J. Synchrotron Radiat. 21, 856 (2014).

[4] M. Borland, G. Decker, L. Emery, V. Sajaev, Y. Sun, and A. Xiao, J. Synchrotron Radiat. 21, 912 (2014).

[5] G. Grübel, A. Madsen, and A. Robert, in Soft Matter Characterization (Springer, New York, 2008) pp. 953-995.

[6] O. G. Shpyrk, J. Synchrotron Radiat. 21, 1057 (2014).

[7] J. Miao, P. Charalambous, J. Kirz, and D. Sayre, Nature (London) 400, 342 (1999).

[8] L. W. Whitehead, G. J. Williams, H. M. Quiney, D. J. Vine, R. A. Dilanian, S. Flewett, K. A. Nugent, A. G. Peele, E. Balaur, and I. McNulty, Phys. Rev. Lett. 103, 243902 (2009).

[9] P. Thibault, M. Guizar-Sicairos, and A. Menzel, J. Synchrotron Radiat. 21, 1011 (2014).

[10] C. G. Schroer and G. Falkenberg, J. Synchrotron Radiat. 21, 996 (2014). 
[11] M. D. de Jonge, C. G. Ryan, and C. J. Jacobsen, J. Synchrotron Radiat. 21, 1031 (2014).

[12] O. Chubar, L. Berman, Y. S. Chu, A. Fluerasu, S. Hulbert, M. Idir, K. Kaznatcheev, D. Shapiro, Q. Shen, and J. Baltser, Proc. SPIE Int. Soc. Opt. Eng. 8141, 814107 (2011).

[13] A. Singer and I. A. Vartanyants, Proc. SPIE Int. Soc. Opt. Eng. 8141, 814106 (2011).

[14] M. Idir, M. Cywiak, A. Morales, and M. H. Modi, Opt. Express 19, 19050 (2011).

[15] A. Prodi, E. Knudsen, P. Willendrup, and S. Schmitt, Proc. SPIE Int. Soc. Opt. Eng. 8141, 814108 (2011).

[16] X. Shi, R. Reininger, M. S. del Rio, and L. Assoufid, J. Synchrotron Radiat. 21, 669 (2014).

[17] K.-J. Kim, AIP Conf. Proc. 184, 565 (1989).

[18] K.-J. Kim, Nucl. Instrum. Methods Phys. Res., Sect. A 246, 71 (1986).

[19] G. Geloni, E. Saldin, E. Schneldmiller, and M. Yurkov, Nucl. Instrum. Methods Phys. Res., Sect. A 588, 463 (2008).

[20] L. Mandel and E. Wolf, Optical Coherence and Quantum Optics (Cambridge University Press, Cambridge, England, 1995).

[21] J. Bahrdt, Phys. Rev. ST Accel. Beams 10, 060701 (2007).

[22] O. Chubar, P. Elleaume, S. Kuznetsov, and A. Snigirev, in Optical Design and Analysis Software II, edited by
R. C. Juergens, Proceedings of SPIE 4769, 145 (2002), http://dx.doi.org/10.1117/12.481182.

[23] O. Chubar, Infrared Phys. Technol. 49, 96 (2006).

[24] I. V. Bazarov, Phys. Rev. ST Accel. Beams 15, 050703 (2012).

[25] T. Tanaka, Phys. Rev. ST Accel. Beams 17, 060702 (2014).

[26] G. Geloni, V. Kocharyan, and E. Saldin, arXiv:1407.4591.

[27] M. J. Bastiaans, J. Opt. Soc. Am. A 3, 1227 (1986).

[28] K.-J. Kim and R. G. Littlejohn, in Proceedings of the Particle Accelerator Conference, Dallas, TX, 1995 (IEEE, New York, 1996), p. 3358.

[29] F. Gori, Opt. Commun. 34, 301 (1980).

[30] A. Starikov and E. Wolf, J. Opt. Soc. Am. 72, 923 (1982).

[31] R. Coisson and S. Marchesini, J. Synchrotron Radiat. 4, 263 (1997).

[32] I. A. Vartanyants and A. Singer, New J. Phys. 12, 035004 (2010).

[33] L. N. Trefethen and J. A. C. Weideman, SIAM Rev. 56, 385 (2014).

[34] B. Y. Rubinstein, Simulation and the Monte Carlo Method (Wiley \& Sons, New York, 1981).

[35] J. Halton, Commun. Assoc. Comput. Machinery 7, 701 (1964).

[36] G. E. P. Box and M. E. Muller, Ann. Math. Stat. 29, 610 (1958). 